\title{
Accurate interface normal and curvature estimates on three-dimensional unstructured non-convex polyhedral meshes
}

\author{
Christopher B. Ivey ${ }^{\mathrm{a}, *}$, Parviz Moin ${ }^{\mathrm{a}}$ \\ ${ }^{a}$ Center for Turbulence Research, Stanford University, Stanford, CA 94305, USA
}

\begin{abstract}
This paper presents a framework for extending the height-function technique for the calculation of interface normals and curvatures to unstructured non-convex polyhedral meshes with application to the piecewise linear interface calculation volume-of-fluid method. The methodology is developed with reference to a collocated node-based finite-volume two-phase flow solver that utilizes the mediandual mesh, requiring a set of data structures and algorithms for non-convex polyhedral operations, namely: truncation of a polyhedron by a plane, intersection of two polyhedra, joining of two convex polyhedra, volume enforcement of a polyhedron by a plane, and volume fraction initialization by a signed-distance function. By leveraging these geometric tools, a geometric interpolation strategy for embedding structured height-function stencils in unstructured meshes is developed. The embedded height-function technique is tested on surfaces with known interface normals and curvatures, namely cylinder, sphere, and ellipsoid. Tests are performed on the median duals of a uniform cartesian mesh, a wedge mesh, and a tetrahedral mesh, and comparisons are made with conventional methods. Across the tests, the embedded height-function technique outperforms contemporary methods and its accuracy approaches the accuracy that the traditional height-function technique exemplifies on uniform cartesian
\end{abstract}

\footnotetext{
* Corresponding author: Phone: 757-570-2369

Email addresses: civey@stanford.edu (Christopher B. Ivey), moin@stanford.edu (Parviz Moin)
}

Preprint submitted to Journal of Computational Physics

July 12, 2015 
meshes.

Keywords: Two-phase flow, Volume of fluid, Height function, Unstructured grid, Curvature, Non-convex polyhedron

\section{Introduction}

The volume-of-fluid (VOF) method is one of the most widely used formulations to simulate interfacial and free-surface flows [1]. In this method, the interface evolution is implicitly tracked using a discrete function, $F$, representing the volume fraction of the tagged fluid within a cell of the computational mesh. $F$ is a discretized version of the fluid marker function, $f$, that is constant in each phase, jumps at the interface from 0 to 1 , and follows the scalar convection equation,

$$
\frac{\partial f}{\partial t}+\vec{v} \cdot \nabla f=0
$$

where $\vec{v}$ is the velocity vector.

The piecewise-linear interface calculation (PLIC) has become the standard interface representation within the VOF community [2]. PLIC-VOF methods describe the interface by a series of disconnected planes, each oriented by a unit normal, $\hat{n}$, and positioned by a constant, $C$, such that $\hat{n} \cdot \vec{x}+C=0$. Two key steps in any PLIC representation include volume truncation by a fixed plane, i.e. determination of $F$ given $\hat{n}$ and $C$, and volume enforcement by a movable plane, i.e. determination of $C$ given $\hat{n}$ and $F$. The importance of the volume truncation and enforcement operations has led researchers to develop analytic and geometric tools to expedite computations for rectangular and hexahedral elements [3], for triangular and tetrahedral elements [4], and for convex polyhedral elements [5]. In this paper, we extend the class of geometric tools to non-convex polyhedral meshes in order to implement the PLIC-VOF method in a collocated node-based finite-volume flow solver [6]. As evidenced by the volume enforcement and truncation operations, estimation of $\hat{n}$ is key to the accuracy of any PLIC-VOF method. 
The equations governing the motion of an unsteady, viscous, incompressible, immiscible two-fluid system are the Navier-Stokes equations, augmented by a localized surface tension force,

$$
\begin{aligned}
\rho\left(\frac{\partial \vec{v}}{\partial t}+\vec{v} \cdot \nabla \vec{v}\right) & =-\nabla p+\nabla \cdot\left(\mu\left[\nabla \vec{v}+\{\nabla \vec{v}\}^{T}\right]\right)-\sigma \kappa \hat{n} \delta_{S}, \\
\nabla \cdot \vec{v} & =0 \\
\kappa & =\nabla \cdot \hat{n}
\end{aligned}
$$

where, $\rho$ is the density, $p$ is the pressure, $\mu$ is the viscosity, $\sigma$ is the surface tension coefficient, $\kappa$ is the interface curvature, and $\delta_{S}$ is the Dirac Delta function localized to the surface $S$. As evidenced by Eq. 2, in addition to accurately estimating $\hat{n}$, the PLIC-VOF framework also needs to calculate the rate at which $\hat{n}$ turns along the interface, i.e. the curvature, $\kappa$.

Determination of $\hat{n}$ and $\kappa$ in the VOF method is problematic due to the discontinuous nature of $F$. Nevertheless, various algorithms to calculate $\hat{n}$ and $\kappa$ have been proposed. The traditional Parker-Youngs (PY) method [7] uses simple difference formulas to calculate gradients in $F$ for the estimation of $\hat{n}$. The method has been implemented on nonorthogonal [8] and unstructured meshes [9]; however, the PY method is at most first-order accurate because $\hat{n}$ for a rectilinear interface is not calculated exactly, a necessary condition for second-order accuracy [10]. The least-squares fit procedure $[11,12]$ is more accurate than the PY method and has been extended to unstructured meshes [13]; however, it too does not satisfy the necessary condition for a second-order method. Several second-order methods for estimating $\hat{n}$ have been proposed, namely the leastsquares volume-of-fluid interface reconstruction algorithm (LVIRA) and the efficient least-squares volume-of-fluid interface reconstruction algorithm (ELVIRA) for structured grids [10], and the geometric least-squares (GLS) method for unstructured grids [14], each able to reconstruct a rectilinear interface exactly. LVIRA orients $\hat{n}$ such that the discrepancy in $F$ from using the calculated linear interface over a neighborhood of cells is minimized in the least-squares sense. The procedure requires costly geometric iterations in which volume enforcement and volume truncation steps must be performed for each cell. ELVIRA bypasses 
the iterations by selecting $\hat{n}$ amongst a set of candidates constructed from the centered, backward, and forward estimates in each direction. The GLS method follows the procedure of LVIRA, requiring geometric iterations within an unstructured framework - a prohibitively costly procedure. A well-known noniterative method for estimating $\hat{n}$ is the height-function (HF) technique. In the HF method, $F$ is integrated in the cartesian direction closest to $\hat{n}$ (approximated with a simpler method) to calculate a height, $H$. Slopes of a local $H$ distribution in the other two cartesian directions are used to correct $\hat{n}[15,16,17]$. The HF method was shown to be second-order accurate with proper handling for particular alignments of the interface with respect to the grid lines $[18,19]$. In two dimensions, the method was extended to nonuniform rectangular grids [20] and, by adapting the definition of $H$ and using a least-squares fit, to unstructured rectangular/triangular grids [21], both exhibiting second-order convergence in $\hat{n}$.

As shown in Eq. 2, $\kappa$, requires higher differentiability than that of $\hat{n}$. To address the lack of differentiability of $F$, various methods have been posited to calculate $\kappa$. In the convolved VOF $(\mathrm{CV})$ method, $F$ is convolved with a smoothing kernel to provide a smoothed-out distribution from which the second derivates can be calculated $[15,22]$. The reconstructed-distance function (RDF) method builds a signed-distance function away from the interface to provide a smooth field from which $\kappa$ can be calculated [15]. The RDF technique was extended to unstructured rectangular/triangular meshes [23]. Both the CV and RDF methods have shown lack of convergence under refinement on structured [15] and unstructured [21] meshes. In addition to the calculation of $\hat{n}$, the HF method has been used to calculate $\kappa$ [15], demonstrating second-order accuracy over a series of canonical test problems on uniform cartesian meshes $[18,24,25]$. In two dimensions, the calculation of $\kappa$ with the HF method was extended to nonuniform rectangular grids without loss of the second-order convergence [20]. The HF technique was extended to two-dimensional unstructured rectangular/triangular grids [21]; however, the reframed definition of $H$ required quadric fitting to calculate $\kappa$, and the method was less than first-order accurate. 
An HF method with $\hat{n}$-aligned columns of variable $H$ was shown to improve the $\kappa$ calculation on coarse meshes; however, it required a neighborhood search step to compute intersections with the mesh and the columns [26]. The HF method was also adapted to quad and octree discretizations [27], where cartesian stencils of varying $H$ (in addition to parabolic fitting for stencils with ill-defined $H$ ) were used to obtain second-order accuracy. To the best of our knowledge, the HF technique for calculating $\hat{n}$ and $\kappa$ has not been extended to three-dimensional unstructured meshes. Furthermore, a convergent method to calculate $\kappa$ on unstructured meshes has not been published.

In this paper, we extend the HF technique for estimating $\hat{n}$ and $\kappa$ to threedimensional unstructured non-convex polyhedral meshes. The method embeds structured HF stencils in the unstructured mesh and interpolates the mesh $F$ data to the stencils to construct the $H$ distribution; as such, it will be called the embedded height-function (EHF) technique in the rest of the article. The EHF technique differs from the unstructured HF method [21] and the $\hat{n}$-aligned HF method [26] in that it uses the traditional $H$ distribution on cartesian stencils to calculate numerical derivates in an attempt to reclaim the second-order accuracy of the structured HF method in the calculation of $\hat{n}$ and $\kappa$. The structure of the paper is as follows. Section 2 presents the EHF technique, where Sec. 2.1 reviews the traditional structured HF technique, Sec. 2.2 discusses the construction of embedded stencils, and Sec. 2.3 presents two geometric methods for interpolating $F$ from the mesh to the EHF stencils. Section 3 develops the geometric tools necessary to perform PLIC-VOF and EHF computations on nonconvex polyhedral grids. Section 4 demonstrates the performance of the EHF technique, as compared to the contemporary methods, on canonical surfaces with known $\hat{n}$ and $\kappa$. 


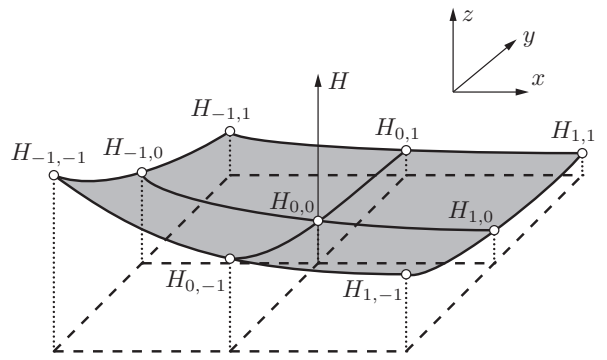

Figure 1: $3 \times 3$ stencil for the calculation of $\hat{n}$ and $\kappa$ from $H$. The interface is represented by a dark gray region bordered by a solid black line. The $H$ 's are visualized using a dotted black line bounded above by an empty circle. The base of the $H$ 's is outlined in a dashed black line.

\section{Interface normal and curvature calculation}

\subsection{Overview of the height-function technique on a structured grid}

The HF method has been shown to be second-order convergent in $\hat{n}$ and $\kappa$ numerically $[15,19,24]$. The second-order accuracy was corroborated analytically provided that the function describing the interface could be approximated to second order with $H$ [18]. The constraint requires that the fourth derivative of the function is bounded and, for the mean value theorem to hold, that the interface crosses the HF column. To reconstruct $H$ from the $F$ data, the mean value theorem requires that the series of cells in the HF column are bounded by a full cell, $F=1$, and an empty cell, $F=0$. The algorithm described herein was designed to meet such constraints using fixed wide symmetric stencils. The static nature of stencils aids in the development of a cost-effective embedding procedure required by the EHF method (see Sec. 2.2).

This section provides an overview of the HF method for calculating the normal and curvature on uniform cartesian meshes with a focus on a $3 \times 3 \times$ 7 stencil aligned such that the maximum dimension is in the HF direction. Consider an interfacial cell $(i, j, k)$, where $0<F<1$, in which the absolute value of the $z$ component of the $\hat{n}, n_{z}$, is largest (assume $\hat{n}$ is known and points outward). Using a $3 \times 3$ stencil centered at the cell $(i, j, k)$ on the $x y$ plane, as 
depicted in Fig. 1, a local distribution of $H$ is calculated as

$$
H_{r, s}=\sum_{t=-t_{\text {down }}}^{t_{\text {up }}} F_{i+r, j+s, k+t}^{*} \Delta z \text {, for } r=-1,0,-1 \text { and } s=-1,0,1,
$$

where $t_{\text {down }}$ and $t_{u p}$ are adaptively adjusted from 1 to $3, \Delta z$ is the cell size in the $z$ direction, and $F^{*}$ is a modified distribution of $F$ that is forced to follow a local monotonic variation along the $z$ direction. The stencil is adapted as suggested by Hérnandez et al. [28]. $t_{u p}$ is gradually increased up to a maximum of 3 cells as long as

$$
\operatorname{sign}\left(n_{z}\right)\left(\alpha_{k+t_{u p}}-\alpha_{k+t_{u p}-1}\right)<0 \text { and } 0<\alpha_{k+t_{u p}}<9,
$$

where $\alpha_{k}=\sum_{r=-1}^{1} \sum_{s=-1}^{1} F_{i+r, j+s, k} . t_{d o w n}$ is adjusted similarly. The stencil adaptation guarantees that the total $F$ across each level of the stencil, $\alpha$, is monotonic in the HF direction. $F^{*}$ satisfies local monotonicity across the HF direction by the following modification proposed by López et al. [24]:

$$
\begin{aligned}
& F_{i+r, j+s, k+t}^{*}=\frac{1}{2}\left(1-\operatorname{sign}(t) \operatorname{sign}\left(n_{z}\right)\right), \\
& \quad \quad \text { if } \operatorname{sign}\left(n_{z}\right)\left(F_{i+r, j+s, k+t}-F_{i+r, j+s, k+t+1}\right)<0 \text { for } t=-1, \ldots,-t_{d o w n} \\
& \quad \quad \text { or } \operatorname{sign}\left(n_{z}\right)\left(F_{i+r, j+s, k+t}-F_{i+r, j+s, k+t-1}\right)>0 \text { for } t=1, \ldots, t_{u p} . \\
& F_{i+r, j+s, k+t}^{*}=F_{i+r, j+s, k+t} \text { otherwise. }
\end{aligned}
$$

From the local $H$ distribution, $\hat{n}$ and $\kappa$ of the interface at cell $(i, j, k)$ are determined as

$$
\begin{aligned}
& \hat{n}=-\frac{\left\langle H_{x} H_{y}-\operatorname{sign}\left(n_{z}\right)\right\rangle^{\top}}{\sqrt{1+H_{x}^{2}+H_{y}^{2}}}, \\
& \kappa=-\frac{H_{x x}\left(1+H_{y}^{2}\right)+H_{y y}\left(1+H_{y}^{2}\right)-2 H_{x y} H_{x} H_{y}}{\left(1+H_{x}^{2}+H_{y}^{2}\right)^{3 / 2}},
\end{aligned}
$$

where $H_{x}$ and $H_{y}$ are the partial derivatives of $H$ in the $x$ and $y$ directions, respectively.

The partial derivatives of $H$ over the $3 \times 3$ stencil can be calculated, to second-order accuracy, with the smoothed central difference formulas [24, 25] 
written as

$$
\begin{aligned}
H_{x}(\gamma) & =\left[\gamma\left(H_{1,1}-H_{-1,1}\right)+\left(H_{1,0}-H_{-1,0}\right)\right. \\
& \left.+\gamma\left(H_{1,-1}-H_{-1,-1}\right)\right] /(2 \Delta x(1+2 \gamma)) \\
H_{x x}(\gamma) & =\left[\gamma\left(H_{1,1}-2 H_{0,1}+H_{-1,1}\right)+\left(H_{1,0}-2 H_{0,0}+H_{-1,0}\right)\right. \\
& \left.+\gamma\left(H_{1,-1}-2 H_{0,-1}+H_{-1,-1}\right)\right] /\left(\Delta x^{2}(1+2 \gamma)\right) \\
H_{x y} & =\left[\left(H_{1,1}-H_{1,-1}\right)-\left(H_{-1,1}-H_{-1,-1}\right)\right] /(4 \Delta x \Delta y)
\end{aligned}
$$

where $\gamma$ is a smoothing coefficient, and $\Delta x$ and $\Delta y$ are the grid spacings in the $x$ and $y$ directions, respectively. The partial derivatives in the $y$ direction are calculated similarly. As shown in Eq. 7, the partial derivatives in a direction are potentially smoothed along the other direction in the plane. $\gamma$ is prescribed to improve the $\hat{n}$ and $\kappa$ computation by averaging out the errors in $H$ (see discussion in Sec. 4.3). By formulation, the smoothing procedure can only be applied to three-dimensional calculations. Local osculating spheres have also been implemented to further correct $H$ [25]; however, this correction was not invoked here to agglomerate all secondary corrections into $\gamma$, simplifying the analysis.

Figure 2 details a two-dimensional numerical example wherein the computed $\hat{n}$ and $\kappa$ accuracy of the stencil adjustment (Fig. 2b) and the local monotonicity correction (Fig. 2c) are compared to that of the full, unmodified HF stencil (Fig. 2a). As can be discerned from the example, the adjusted stencil and the modified $F$ distribution address the loss of accuracy when multiple interfaces are within the HF stencil. The $\hat{n}$ and $\kappa$ of cell $(i, j, k)$ are determined using Eq. 6 if $z_{k-1 / 2}<H_{0,0}<z_{k+1 / 2}$, where $z_{k-1 / 2}$ and $z_{k+1 / 2}$ are the heights of the lower and upper faces of cell $(i, j, k)$ from the bottom face of the lowest HF stencil cell $\left(i, j, k-t_{\text {down }}\right)$. When this constraint is not satisfied, like in cell $(i, j+1)$ of Fig. 2c, the interface crosses multiple cells along the middle HF column, degrading the accuracy of this method $[18,19,24]$. For the interface cells, $0<F<1$, that do not satisfy this criterion, $\hat{n}$ and $\kappa$ are determined through an interpolation of the neighboring interface cells that do satisfy this criterion. If the interface cell is isolated, meaning that there are no neighbors satisfying the HF interface 
criterion, $\hat{n}$ and $\kappa$ have to be determined through auxiliary methods that do not involve HF (the PY and CV methods are used here, respectively).

An alternative approach to using fixed wide symmetric stencils is to build asymmetric stencils, adapted to the geometry such that each column of the stencil is independently extended until it is bounded by a full and empty cell [27]. If any of the columns cannot be extended such that they are bound by a full and empty cell, the interface is declared under-resolved and an alternative approach is used. In such scenarios, Popinet [27] used parabolic fitting to calculate curvature. Owkes et al. [26] used $\hat{n}$-aligned columns of varying $H$ to get a more accurate estimate of curvature for under-resolved features. The asymmetric stencils naturally meet the analytic constraints for a second-order estimate of curvature without the $F$ modification used here and readily identify under-resolved features. This approach was not taken because the time-consuming embedding procedure (see Sec. 2.2), required by the EHF method, can be performed as a preprocessing step when the stencils are statically allocated. Without properly accommodating under-resolved features, the utilized definition of the HF will potentially result in a reduction of accuracy at coarse resolutions; however, it will not impact the asymptotic convergence of the method.

\subsection{Construction of height-function stencils on an unstructured grid}

Our collocated node-based finite-volume flow solver [6] creates node-based volumes by tessellating each cell of the primal mesh into sub-tetrahedra, each composed of a node, a face center, an edge center, and a cell center, and agglomerating the sub-tetrahedra associated with the node together to form, in general, a non-convex polyhedron. The node volumes comprise the median-dual mesh. An example of a two-dimensional median-dual mesh, composed of nonconvex polygons, formed from a primal mesh composed of convex polygons, is shown in Fig. 3a. As noted in Sec. 2, the construction of the HF stencil maps requires a significant amount of time, which, in a parallel setting, is exacerbated by the need to construct parallel communicators specialized for the embedded HF stencils. Therefore, it is advantageous to use fixed wide symmetric sten- 

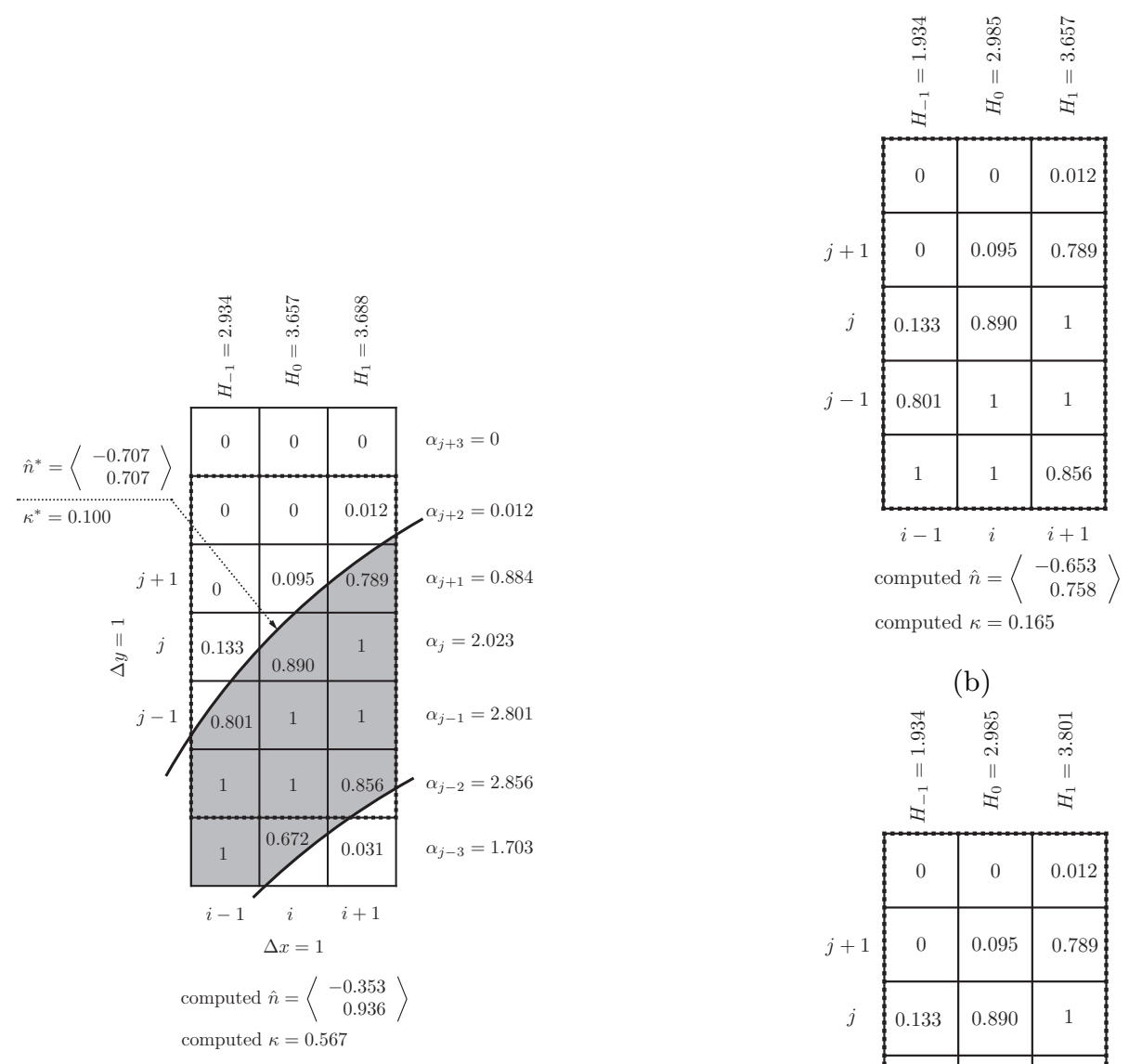

(b)

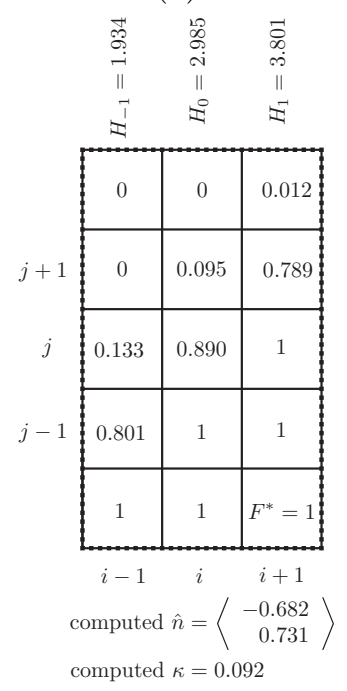

(c)

Figure 2: Calculation of $\hat{n}$ and $\kappa$ in two dimensions using (a) the standard HF stencil, (b) the adjusted HF stencil, and (c) the adjusted HF stencil with the modified $F$ distribution. The exact normal, $\hat{n}^{*}$, is equal to $\langle-11\rangle^{\top} / \sqrt{(2)} \approx\langle-0.7070 .707\rangle^{\top}$ and the exact curvature, $\kappa^{*}$, is equal to 0.1 . The computed $\hat{n}$ and $\kappa$ values are based on the respective $H$ 's of the HF stencil. The tagged fluid is indicated by a dark gray region borded by a thick solid black line in (a) and the adjusted stencil is outlined by a thick dashed black line in (a,b,c). 
cils and to perform the operations necessary to construct the HF stencils (and parallel communicators) once as a preprocessing step.

For each node, the HF stencil is constructed by forming the bounding box of the node volume and shifting it in each coordinate direction in increments of its respective length to fill a $(3 \times 7 \times 7) \cup(7 \times 3 \times 7) \cup(7 \times 7 \times 3)$ stencil. The bounding box for a point set is defined to be the right rectangular prism with the smallest volume within which all points lie. To construct the mapping from the mesh to the stencils at the beginning of the simulation, each stencil encompasses the $x, y$, and $z$ stencils of maximum column height $\left(t_{u p}=t_{\text {down }}=3\right)$, ensuring the availability of any possible subset of the stencil required by Eq. 3 to construct the local $H$ distribution. Each node has its own HF stencil to ensure that the $\hat{n}$ and $\kappa$ estimates for the center bounding box of the HF stencil directly correspond to the node. In other words, if a right rectangular prism is constructed by extruding the base of the embedded HF stencil center column in the HF direction to a height $H$ and the right rectangular prism intersects with the center stencil-unit bounding box, then it also intersects with the node volume. Under this construction, by performing the algorithm in Sec. 2.1 on the stencil, the mean value theorem will hold for the node volume. Unfortunately, a single auxiliary uniform cartesian mesh, similar to that used by the balanced-force refined level-set grid method [29], could not satisfy the constraint placed by the mean value theorem. If stored properly, each HF stencil needs only $3 \cdot(3 \cdot 3 \cdot 7)-2 \cdot(3 \cdot 3 \cdot 3)=135$ stencil units in three dimensions and $2 \cdot(3 \cdot 7)-1 \cdot(3 \cdot 3)=33$ stencil units in two dimensions, where a given stencil unit, $u$, can be indexed by the node, $p$, and the local cartesian indices, $(i, j, k)$ : $u \leftarrow[p,(i, j, k)]$. Each stencil unit needs to store the associated minimum and maximum vertex coordinates of the bounding box, a list of nodes whose volumes intersect with the bounding box, and the intersection volumes of each of these nodes with the bounding box. Storing the polyhedral representation of the intersection between the node volume and the bounding box would be prohibitively memory intensive.

In order to efficiently find all the intersections, the alternating digital tree 
(ADT) search algorithm of [30] is employed. Bounding boxes of the node volumes are used to build the tree structure. Given the ADT and an HF stencilunit bounding box, the node volumes whose bounding boxes intersect with the HF stencil-unit bounding box can be efficiently found. For the node-volume bounding boxes that intersect with the HF stencil-unit bounding box, the node volumes are checked for intersection. If the node volume and the HF stencil unit are found to intersect, the node index and intersection volume are added to the list of nodes. Once all the intersections are found between the mediandual node volumes and the stencils, the intersection indices and volumes for the stencil-units can be packaged tightly into arrays using compressed-row storage (CSR).

Figure $3 \mathrm{~b}$ shows an example of a two-dimensional stencil created from the bounding box of a node volume of the median-dual mesh constructed in Fig. 3a. In a parallel setting, the embedded stencil can reside on multiple processors, which need not be the same as the unstructured stencils used for differentiating the properties on the median-dual mesh. An example partition is shown to the right of Figs. 3a and 3b, where the processor boundary separates cells in the primal mesh, cutting through the node volumes.

\subsection{Interpolation of volume fraction data from unstructured grid to stencils}

To perform the calculation in Sec. 2.1, the HF stencils need to be populated with $F$ data interpolated from the median-dual mesh. As described in Sec. 2.2 , each stencil unit has an associated bounding box and a list of nodes that intersect the bounding box with their respective intersection volumes. From the information available, two simple geometric interpolation schemes can be constructed.

The simplest method to interpolate $F$ to a single HF stencil unit is to take a weighted average of the $F$ data from the nodes it intersects, where the weight is determined by the intersection volume of the node with the stencil-unit bounding box. The intersection volume-weighted (IVW) interpolation follows the 


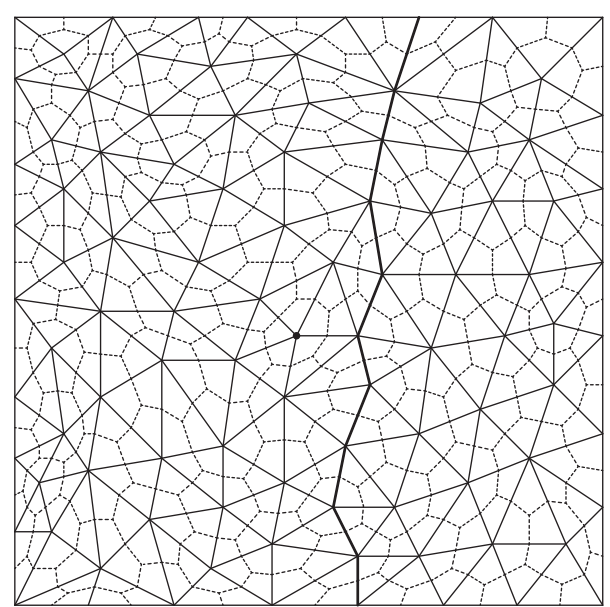

(a)

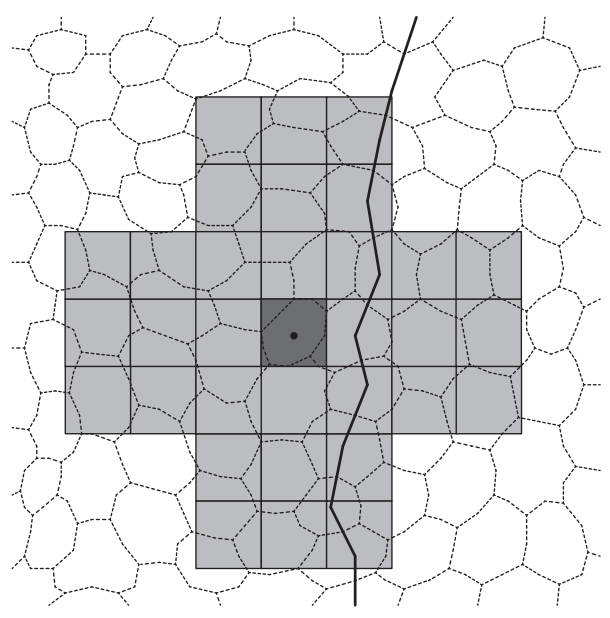

(b)

Figure 3: HF stencil embedded in median-dual mesh. The primal mesh is outlined with a solid black line in (a) and the associated median-dual mesh is outlined with a dashed black line in $(a, b)$. The node of interest is indicated by a filled black circle and the processor boundary is indicated by a thick solid black line in (a,b). The central bounding box of the node volume of interest is indicated by a dark gray region borded by a solid black line, with the associated HF stencil indicated by a light gray region borded by a solid black line, in (b). 
update rule

$$
F_{u}=\frac{\sum_{p \epsilon\{n o\} \cap u} V_{u, p}^{\cap} F_{p}}{V_{u}},
$$

where $F_{u}$ is the volume fraction of stencil-unit $u,\{n o\}$ is the set of all nodes, $\{n o\} \cap u$ is the set of nodes that intersect $u$ 's stencil-unit bounding box, $F_{p}$ is the volume fraction of node $p, V_{u}$ is the volume of $u$ 's stencil-unit bounding box, and $V_{u, p}^{\cap}$ is the intersection volume of $p$ 's node volume and $u$ 's stencil-unit bounding box. Figure 4 illustrates the interpolation scheme for a single stencil unit. IVW interpolation provides a first-order approximation to $F_{u}$; however, except for $F_{p}$, all the required information needs to be calculated once only at the start of the calculation, making it quite efficient over the course of the simulation (note that $V_{u}$ is the same for all units in a given HF stencil). Once $F_{u}$ is determined for every HF stencil unit, $\hat{n}$ and $\kappa$ can be calculated using Eqs. 3-7.

A higher-order interpolation scheme for $F_{u}$ can be constructed by utilizing the PLIC information from the list of intersected nodes. To interpolate using the PLIC reconstruction, $\hat{n}$ needs to be predetermined: e.g., an unstructured version of the PY method [9]. Then the planar interface constant, $C$, needs to be determined through the volume-enforcement operation using $\hat{n}$ and $F$. From the PLIC information a more accurate approximation to $F_{u}$ can be found,

$$
F_{u}=\frac{\sum_{p \epsilon\{n o\} \cap u} V\left(\mathcal{H}_{p}^{\mathrm{PLIC}} \cap \mathcal{P}_{p} \cap \mathcal{P}_{u}\right)}{V_{u}}
$$

where $V(\cdot)$ is the volume operator, $\mathcal{H}_{p}^{\mathrm{PLIC}}$ is the half-space formed behind the PLIC plane (in the $-\hat{n}$ direction) of node $p, \mathcal{P}_{p}$ is the polyhedral representation of $p$ 's node volume, and $\mathcal{P}_{u}$ is the polyhedral representation of the $u$ 's stencilunit bounding box. $\mathcal{H}_{p}^{\mathrm{PLIC}} \cap \mathcal{P}_{p}$ is the polyhedron formed by truncating $\mathcal{P}_{p}$ by the PLIC plane. PLIC, along with an accurate estimate of $\hat{n}$, provides a secondorder approximation to the fluid interface [19], yielding a second-order accurate $F_{u}$. The added accuracy is at the cost of an added volume-enforcement step to reconstruct the PLIC interface. Furthermore for each $p \epsilon\{n o\} \cap u$, interpolation requires a polyhedron-plane truncation and a polyhedron-polyhedron intersection operation to determine $\mathcal{H}_{p}^{\mathrm{PLIC}} \cap \mathcal{P}_{p} \cap \mathcal{P}_{u}$. As mentioned in Sec. 2.2, $\mathcal{P}_{p} \cap \mathcal{P}_{u}$ 


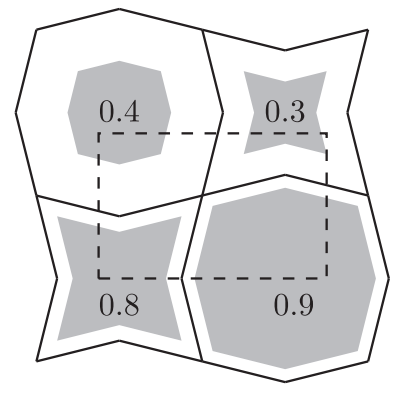

(a)

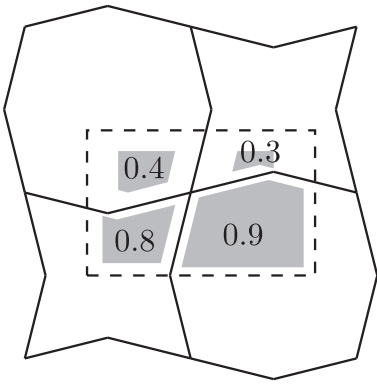

(b)

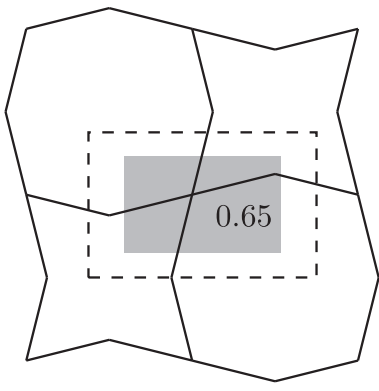

(c)

Figure 4: IVW interpolation of $F_{u}$ of the stencil-unit bounding box $u$ from the $F_{p}$ of the intersected node volumes $p$. The $F_{p}$ in (a) are used to compute the volume contribution of $p$ to $u, F_{p} V_{u, p}^{\cap}$, in (b). Each of the volume contributions in (b), $F_{p} V_{u, p}^{\cap}$, are summed and divided by $u$ 's volume, $V_{u}$, to get the $F_{u}$ in (c). The median-dual mesh is outline by a solid black line in $(a, b, c)$. The stencil-unit bounding box is outlined by a dashed black line in $(a, b, c)$. The volume-fraction is visualized using a light gray region in $(\mathrm{a}, \mathrm{b}, \mathrm{c})$.

could also be stored at the beginning of the simulation, just as $V_{u, p}^{\cap}$, which would remove the polyhedron-polyhedron intersection operation; however, it would create a large memory footprint. For the example of a periodic uniform cartesian mesh composed of $N_{n o}$ nodes, where each $\mathcal{P}_{u}$ intersects only with a single $\mathcal{P}_{p}, 135 \cdot N_{n o}$ non-convex polyhedra would have to be stored. A more general mesh would require even larger storage, so we decided to recalculate $\mathcal{P}_{p} \cap \mathcal{P}_{u}$ during each application of PLIC interpolation. Figure 5 schematically shows the PLIC interpolation procedure in two dimensions. Once $F_{u}$ is determined for the entire HF stencil, $\hat{n}$ can be updated using Eqs. 3-7 and the approximation to $F_{u}$ can be ameliorated by iterating. IVW interpolation, along with Eqs. 3-7, can be used instead of the PY method to get a first estimate of $\hat{n}$ to perform the PLIC interpolation scheme; however, both the PY method and IVW interpolation will provide only a first-order approximation to $F_{u}$, so at least two iterations will be required to get a second-order accurate estimation to $\hat{n}$ and $\kappa$. The accuracy of the interpolation derives from the accuracy of $\hat{n}$ used in the PLIC reconstruction, so different methods to estimate $\hat{n}$ and iteration counts are surveyed in Sec. 4.2 . 


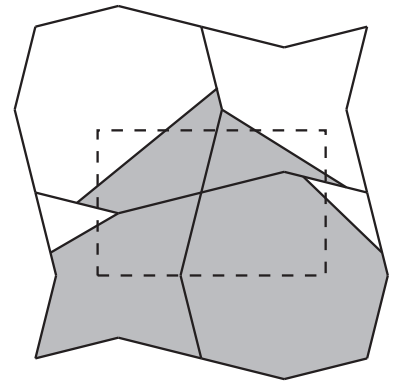

(a)

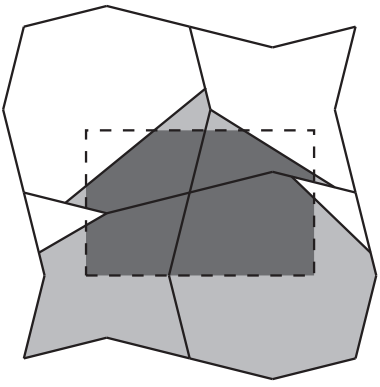

(b)

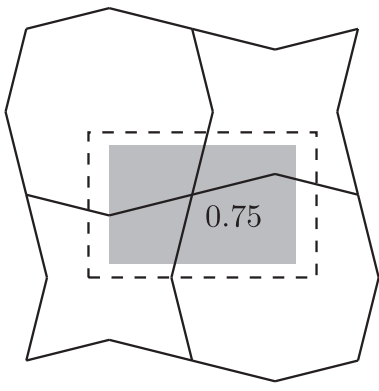

(c)

Figure 5: PLIC interpolation of $F_{u}$ of the stencil-unit bounding box $u$ from the $\mathcal{H}_{p}^{\text {PLIC }}$ of the intersected node volumes $p$. The $\mathcal{H}_{p}^{\mathrm{PLIC}}$ in (a) are used to compute the volume contribution of $p$ to $u, \mathcal{H}_{p}^{\mathrm{PLIC}} \cap \mathcal{P}_{p} \cap \mathcal{P}_{u}$, in (b). Each of the volume contributions in (b), $\mathcal{H}_{p}^{\mathrm{PLIC}} \cap \mathcal{P}_{p} \cap \mathcal{P}_{u}$, are summed and divided by $u$ 's volume, $V_{u}$, to get the $F_{u}$ in (c). The median-dual mesh is outline by a solid black line in $(\mathrm{a}, \mathrm{b}, \mathrm{c})$. The stencil-unit bounding box is outlined by a dashed black line in $(\mathrm{a}, \mathrm{b}, \mathrm{c})$. The PLIC reconstruction is visualized with a light gray region borded by a solid black line in (a,b). The PLIC region in the stencil-unit bounding box is visualized with a dark gray region borded by a solid and dashed black line in (b). The volume-fraction is visualized using a light gray region in (c).

\section{Geometric tools}

As described in Sec. 2.2, the median-dual mesh is composed of non-convex node volumes. To leverage the available geometric tools for convex polyhedra [5], it is advantageous to store non-convex polyhedra as a list of convex polyhedra,

$$
\mathcal{P}=\left\{\mathcal{P}_{1}, \mathcal{P}_{2}, \ldots, \mathcal{P}_{n}\right\}, \quad \mathcal{P}_{i} \text { convex for } i=1,2, \ldots, n,
$$

where $\mathcal{P}$ is the non-convex polyhedron, $\mathcal{P}_{i}$ is the $i$ th convex sub-polyhedron, and $n$ is the number of sub-polyhedra.

The geometric operations for PLIC-VOF on a non-convex polyhedron can be broken down into a composition of operations on the convex decomposition, specifically truncation of a polyhedron by a plane (or, equivalently, the intersection of a polyhedron and the half-space behind a plane) and volume enforcement of a polyhedron with a plane [5]. The EHF method expands the set of required operations to include the intersection of two polyhedra and, 
to minimize the memory footprint of the convex decomposition, the joining of two convex polyhedra. As described in Sec. 2.2, the node volumes are formed from sub-tetrahedra created during the construction of the median-dual, providing a known non-optimal convex decomposition of the node volume. The convex join operation is necessary to reduce the convex set to a more optimal decomposition of the node volume. Note also that geometric VOF convection algorithms (see for example $[9,31,32,33]$ ) also generate polyhedra of known convex decompositions, obviating the need for an expensive and complicated convex-decomposition algorithm. To test the accuracy of the EHF method, it is also necessary to design a second-order accurate method to initialize $F$ from a signed distance function.

The data representation used here follows that of López et. al [5], where each convex-polyhedron stores: the number of nodes, the number of faces, an array of node vertex coordinates, an array of face plane normals, an array of face plane constants, and a map that takes a face-local-node index to a global-node index, which is represented with two arrays using CSR. To reduce the number of reallocations during the geometric operations, the arrays are preallocated using the user-specified number of faces and nodes. Because the polyhedron is not self-intersecting, the number of edges in the polyhedron equals the number of faces plus the number of nodes less two. With CSR, the map arrays scale with the number of edges and faces, respectively, so the specification of the number of faces and nodes is sufficient to allocate all of the arrays in the convex-polyhedron representation. To reduce the memory footprint of the representation, reallocation based on increments of the user-specified number of faces and nodes is used to grow and shrink the convex polyhedron; this requires that the allocated number of faces and nodes is stored in the rest of the convex polyhedron. Efficiency of the representation is highly dependent on the user-specified number of nodes and faces; it was determined that a preallocation of 20 faces and 20 nodes, which could account for most convex polyhedra that occur practically, provided a good balance between memory and time costs. The non-convex polyhedron is represented as an unsorted list of convex polyhedra. If the non-convex poly- 
hedron is to remain unchanged throughout the simulation (e.g. a median-dual volume after the convex join operation), the constituent convex-polyhedra are connected through a fixed-size array.

\subsection{Truncate a non-convex polyhedron by a plane}

The truncation of a non-convex polyhedron by a plane (the intersection of the polyhedron and the half-space behind the plane) is the same as truncating all the convex sub-polyhedra by the plane,

$$
\mathcal{P} \cap \mathcal{H}_{T}=\left\{\mathcal{P}_{1} \cap \mathcal{H}_{T}, \mathcal{P}_{2} \cap \mathcal{H}_{T}, \ldots, \mathcal{P}_{n} \cap \mathcal{H}_{T}\right\}
$$

where $\mathcal{H}_{T}$ is the half-space behind plane $T$.

The convex polyhedron truncation algorithm uses a clipping and capping technique $[5,34]$. The signed distance, $\phi_{v}$, from every vertex, $\vec{x}_{v}$, to the truncation plane, $T$, is calculated using

$$
\phi_{v}=\hat{n}_{T} \cdot \vec{x}_{v}+C_{T}
$$

where $\hat{n}_{T}$ points out of the region to be kept. As such, if $\phi_{v}<0$ for all $\vec{x}_{v}$, then the sub-polyhedron is kept in the list of convex polyhedra, but if $\phi_{v}>0$ for all $\vec{x}_{v}$, then the sub-polyhedron is removed from the list of convex polyhedra, otherwise plane $T$ intersects the sub-polyhedron and the truncated convex subpolyhedron needs to be constructed from the edges intersected by $T$.

An edge is defined as intersected if the sign of $\phi$ in the two nodes bounding the edge is different. The signed distances to the plane are used to locate the intersection vertices, $\vec{x}_{t}$. For example, if an intersected edge is bound by $\vec{x}_{1}$ and $\vec{x}_{2}$, the intersection position along the edge is found using

$$
\vec{x}_{t}=\vec{x}_{1}-\frac{\phi_{1}}{\phi_{2}-\phi_{1}}\left(\vec{x}_{2}-\vec{x}_{1}\right),
$$

where $t$ is the index of the node created by the intersection. The truncated subpolyhedron is formed from the nodes with $\phi<0$ and from the newly generated intersected nodes. 


\subsection{Intersect non-convex polyhedra}

The intersection of two non-convex polyhedra is the union of the set of all possible intersection pairs of their convex sub-polyhedron components,

$$
\begin{aligned}
& \mathcal{P} \cap \mathcal{P}^{\prime}=\left\{\mathcal{P} \cap \mathcal{P}_{1}^{\prime}, \mathcal{P} \cap \mathcal{P}_{2}^{\prime}, \ldots, \mathcal{P} \cap \mathcal{P}_{n}^{\prime}\right\} \\
&=\left\{\left\{\mathcal{P}_{1} \cap \mathcal{P}_{1}^{\prime}, \mathcal{P}_{1} \cap \mathcal{P}_{2}^{\prime}, \ldots, \mathcal{P}_{1} \cap \mathcal{P}_{n}^{\prime}\right\},\right. \\
&\left\{\mathcal{P}_{2} \cap \mathcal{P}_{1}^{\prime}, \mathcal{P}_{2} \cap \mathcal{P}_{2}^{\prime}, \ldots, \mathcal{P}_{2} \cap \mathcal{P}_{n}^{\prime}\right\}, \\
& \ldots, \\
&\left.\left\{\mathcal{P}_{m} \cap \mathcal{P}_{1}^{\prime}, \mathcal{P}_{m} \cap \mathcal{P}_{2}^{\prime}, \ldots, \mathcal{P}_{m} \cap \mathcal{P}_{n}^{\prime}\right\}\right\},
\end{aligned}
$$

where $n$ is number of convex sub-polyhedra that make up non-convex polyhedron $\mathcal{P}^{\prime}$ and $m$ is number of convex sub-polyhedra that make up non-convex polyhedron $\mathcal{P}$, resulting in a non-convex polyhedron formed from potentially $n m$ convex sub-polyhedra. The algorithm is illustrated in the two-dimensional schematic of Fig. 6. In reality, many of the intersections will be empty; however, the intersection of two non-convex polyhedron potentially increases the number of constituent convex sub-polyhedra. To keep the number of convex sub-polyhedra bounded, the convex polyhedron join algorithm (see Sec. 3.3) can be performed after the intersection operation.

The intersection of two convex-polyhedra is the intersection of one of the convex polyhedra with all the half-spaces formed from the planes bounding the other polyhedron,

$$
\mathcal{P}_{i} \cap \mathcal{P}_{j}^{\prime}=\mathcal{H}_{i, 1} \cap \mathcal{H}_{i, 2} \cap \ldots \cap \mathcal{H}_{i, q} \cap \mathcal{P}_{j}^{\prime}
$$

where $\mathcal{H}_{i, r}$ is the half-space behind face $r$ of the $i$ th convex sub-polyhedron of the non-convex polyhedron $\mathcal{P}$ and $q$ is the number of faces of the $i$ th convex sub-polyhedron of the non-convex polyhedron $\mathcal{P}$. The intersection of a convexpolyhedron with a half-space is described in Sec. 3.1. Figure 7 shows a twodimensional schematic of the algorithm.

Before intersecting two non-convex polyhedra, their respective bounding boxes are checked to see if they intersect. If the bounding boxes intersect, then 


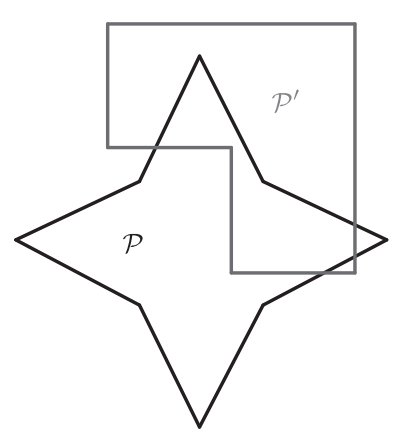

(a)

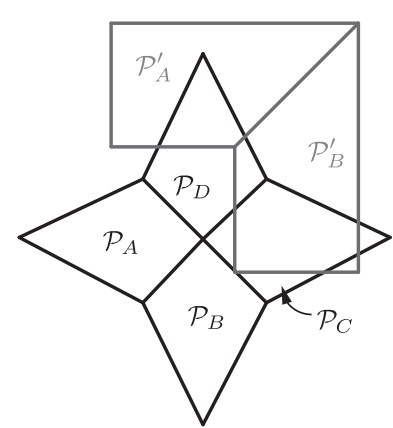

(b)

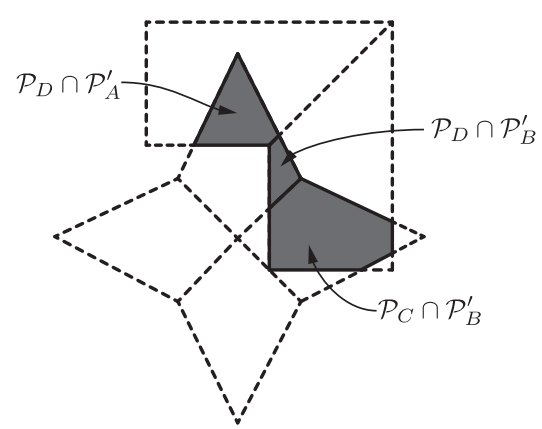

(c)

Figure 6: Intersection of non-convex polygons $\mathcal{P}$ and $\mathcal{P}^{\prime}$, outlined by a solid black line and a dark gray line, respectively (a). The convex sub-polygon representation, $\mathcal{P}=\left\{\mathcal{P}_{A}, \mathcal{P}_{B}, \mathcal{P}_{C}, \mathcal{P}_{D}\right\}$ and $\mathcal{P}^{\prime}=\left\{\mathcal{P}_{A}^{\prime}, \mathcal{P}_{B}^{\prime}\right\}$, outlined by a solid black line and a dark gray line, respectively, is shown in (b). The resulting intersection, $\mathcal{P} \cap \mathcal{P}^{\prime}=\left\{\mathcal{P}_{D} \cap \mathcal{P}_{A}^{\prime}, \mathcal{P}_{D} \cap \mathcal{P}_{B}^{\prime}, \mathcal{P}_{C} \cap \mathcal{P}_{B}^{\prime}\right\}$, is shown in (c), where the intersection region is indicated by a dark gray region borded by a solid black line and the removed region is outlined by a dashed black line.

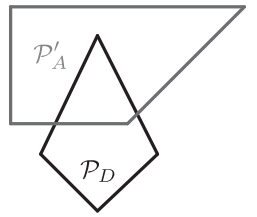

(a)

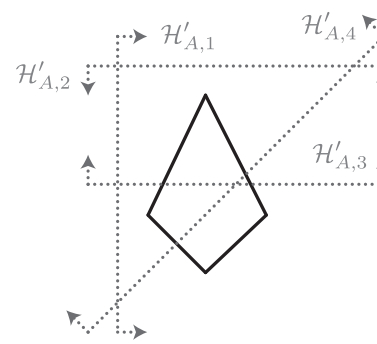

(b)

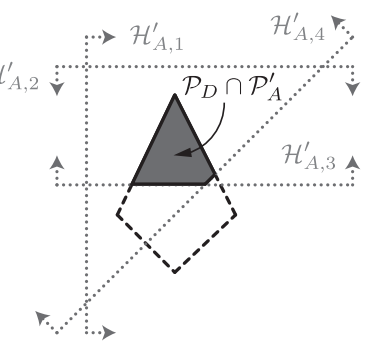

(c)

Figure 7: Intersection of convex polygons $\mathcal{P}_{D}$ and $\mathcal{P}_{A}^{\prime}$ from Fig. 6, outlined by a solid black line and a dark gray line, respectively (a). The conversion of one of the polygons into the half-spaces created from its edges, $\mathcal{P}_{A}^{\prime}=\mathcal{H}_{A, 1}^{\prime} \cap \mathcal{H}_{A, 2}^{\prime} \cap \mathcal{H}_{A, 3}^{\prime} \cap \mathcal{H}_{A, 4}^{\prime}$, is shown in (b), with the half-spaces bordered by a dotted dark gray line. The resulting intersection, $\mathcal{P}_{D} \cap \mathcal{P}_{A}^{\prime}$, is shown in (c), where the intersection region is indicated by a dark gray region bordered by a solid black line and the removed region is outlined by a dashed black line. 


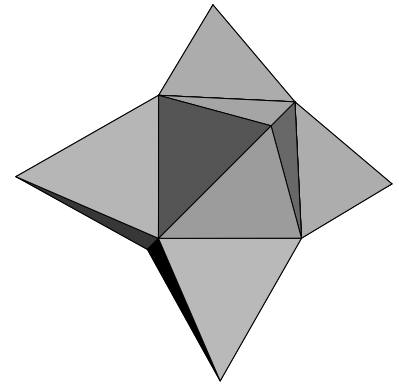

(a)

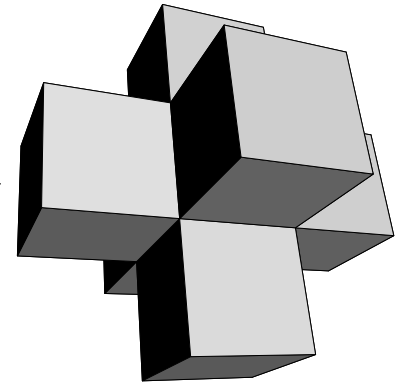

(b)

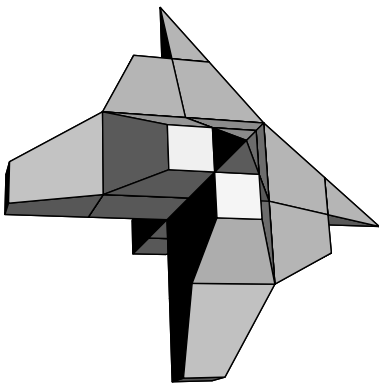

(c)

Figure 8: Intersection between a non-convex polyhedron created from a unit cube centered at $\left[\begin{array}{lll}0 & 0 & 0\end{array}\right]^{\top}$ with square pyramids of unit height attached to each face (a) and a non-convex polyhedron created from a unit cube centered at $\left[\begin{array}{lll}1 / 2 & 1 / 2 & 1 / 2\end{array}\right]^{\top}$ with unit cubes attached to each face (b). The resultant intersection is shown in (c).

the bounding boxes for all possible pairs of their respective convex sub-polyhedra are checked for intersection. The actual intersection is then performed only on the pairs' convex sub-polyhedra whose bounding boxes intersect. Boundingbox intersection checks are fast, so this two-level bounding box check greatly decreases the runtime. Figure 8 shows an example of a three-dimensional intersection operation between two non-convex polyhedra using the algorithm described. The number of sub-polyhedra in Fig. $8 \mathrm{c}$ is greater than those of Figs. $8 \mathrm{~b}$ and $8 \mathrm{a}$, illustrating the need to join adjacent convex sub-polyhedra into a single convex sub-polyhedron.

\subsection{Join convex polyhedra}

As described at the beginning of Sec. 3, the node volumes are composed of many sub-tetrahedra. Further, the non-convex intersection algorithm in Sec. 3.2 can increase the number of sub-polyhedra. To maintain the efficacy of the convex sub-polyhedron description, it is necessary to join convex sub-polyhedra together to form a smaller set of space-filling convex polyhedra.

A locally optimal greedy algorithm [35] is employed here. At each stage of the algorithm, the largest two polyhedra (by volume) that share a face and can be joined to form a convex sub-polyhedron are joined together, removing one 
sub-polyhedron from the list. The actual join involves removing the shared face, merging the resultant coplanar faces, and updating the topological connectivities. The procedure repeats until there is only one convex sub-polyhedron, or no two sub-polyhedra can be joined. The resultant set of convex sub-polyhedra will provide a sub-optimal convex decomposition of the polyhedron (that is not guaranteed to contain the minimum number of convex sub-polyhedra).

Assuming that two convex-polyhedra share a face (the vertex locations are the same), the joining of the two sub-polyhedra will form a convex polyhedron as long as all the edges of the shared face of the resultant polyhedron are not reflex edges [36]. An edge is a reflex edge if the dihedral angle of the two faces that bound the edge is greater than $\pi$. Because each sub-polyhedron is individually convex, the edges not bordering the shared face need not be checked for local convexity.

A simple method to determine whether an edge is a reflex edge is to substitute the vertices of one face bordering the edge into the plane equation (Eq. 12) of the other face. If $\phi<0$ for all points of the face not on the edge, assuming the other face has an outward pointing $\hat{n}$, the points lie in the half-space formed behind the plane of the other, and the edge is not a reflex edge. An equivalent two-dimensional example is illustrated in Fig. 9, where polygons $\mathcal{P}_{A}$ and $\mathcal{P}_{B}$ in Fig. 9a are checked to see if they can be merged into a single convex polygon. Fig. $9 \mathrm{~b}$ shows that the vertex of $\mathcal{P}_{A}$ of the edge connected to the top shared vertex is in the half-space $\mathcal{H}_{B, 1}$ created behind the edge of $\mathcal{P}_{B}$ adjacent to the shared top vertex, indicating that the top shared vertex is convex. Fig. 9c shows that the vertex of $\mathcal{P}_{A}$ of the edge connected to the bottom shared vertex is not in the half-space $\mathcal{H}_{B, 2}$ created behind the edge of $\mathcal{P}_{B}$ adjacent to the shared bottom vertex, indicating the bottom shared vertex is non-convex. Because a vertex of the shared edge is reflexive (non-convex), the joining of $\mathcal{P}_{A}$ and $\mathcal{P}_{B}$ would be non-convex.

The node volumes may be non-convex and may contain many convex subpolyhedra. Figure 10 shows the impact of the algorithm for a single node-volume element from a primal mesh composed of tetrahedral cells. The algorithm re- 


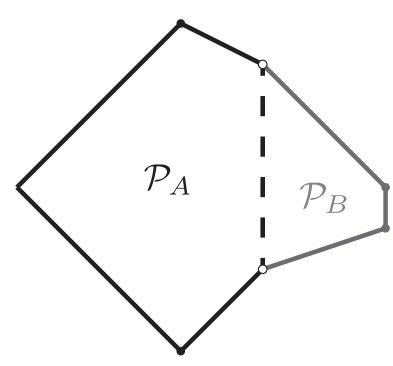

(a)

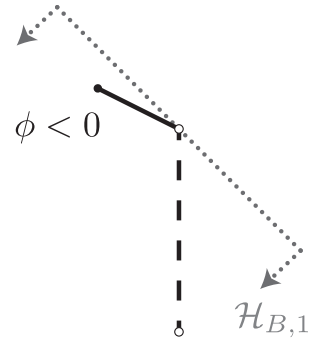

(b)

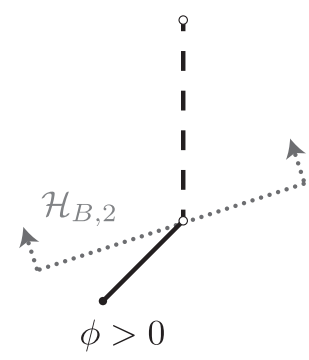

(c)

Figure 9: Convexity check for the joining of two convex polygons, $\mathcal{P}_{A}$ and $\mathcal{P}_{B}$, outlined by a solid black line and a dark gray line (excluding the shared edge), respectively (a). The shared edge is indicated by a dashed black line in $(\mathrm{a}, \mathrm{b}, \mathrm{c})$. The shared vertices are indicated by an empty black circle, while the unshared vertices are shown as a filled black circle in $(\mathrm{a}, \mathrm{b}, \mathrm{c})$. The half-spaces are bordered by a dotted dark gray line in (b,c). (b) shows that the top vertex of the edge shared between $\mathcal{P}_{A}$ and $\mathcal{P}_{B}$ is convex. (c) shows that the bottom vertex of the edge shared between $\mathcal{P}_{A}$ and $\mathcal{P}_{B}$ is non-convex.

duced the number of components from 144 to 20, a substantial reduction in memory and operations.

\subsection{Enforce volume of a non-convex polyhedron by a plane}

Building on the work of López et al. [5], the volume of a non-convex polyhedron, which is the net volume of the set of convex sub-polyhedra, can be parameterized by the constant $C$ of the plane of fixed $\hat{n}$. The resultant volume formula would still be cubic in $C$ and could be solved analytically. However, the analytic solution would require bracketing each convex sub-polyhedron to find the vertices of the original polyhedron that will remain in the truncated polyhedron. Bracketing the solution requires a non-convex polyhedron truncation operation and volume calculation for each vertex of each sub-polyhedron, which is prohibitively costly for the convex decomposition representation of a non-convex polyhedron.

Instead, the non-convex polyhedron-plane truncation operation from Sec. 


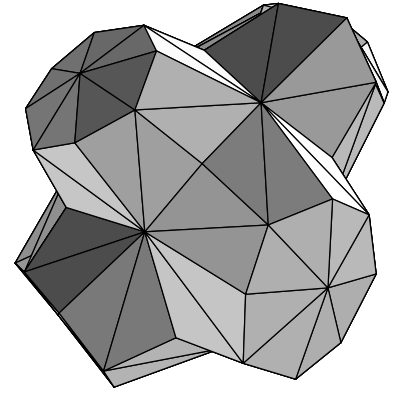

(a)

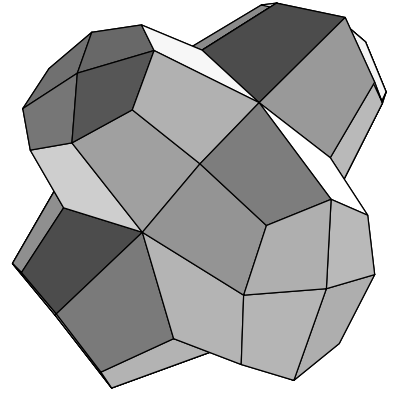

(b)

Figure 10: Convex composition of a median-dual node volume created from a tetrahedral primal mesh. The original decomposition of 144 sub-tetrahedra, created from the median-dual discretization, is shown in (a). The final decomposition of 20 convex sub-polyhedra, created from the convex joining of sub-tetrahedra in the node volume from the greedy algorithm, is shown in (b).

3.1 is wrapped with Brent's method to solve for the truncation plane constant $C$ iteratively. Each iteration consists of copying the set of convex sub-polyhedra, truncating the copy of the set of convex sub-polyhedra with a plane of a prescribed $C$, calculating the net volume of the copy of the set of convex subpolyhedra, and updating $C$ with the error in the calculated volume. Across the tests, Brent's method required $10-30$ iterations to converge the error in the calculated volume fraction to $10^{-12}$. The total number of nodes of the nonconvex polyhedra could be as low as 4 (for an optimal convex composition of a hexahedral element); however, it was usually larger than 100 (160 vertices made up the convex decomposition in Fig. 10b). As the average number of iterations is much smaller than the total number of nodes of the set of convex sub-polyhedra, and therefore the number of non-convex polyhedron truncation operations and volume calculations, it was determined that Brent's method was favorable to an analytic solution.

\subsection{Initialize the volume-fraction by the signed distance}

An important factor that may undermine the accuracy of the calculated interface properties is the initialization of $F$. It is difficult to know a priori the 
$F$ of a node given an analytic description of the surface. A second-order accurate procedure, extending that described by López et al. [24] to unstructured meshes, is developed here.

A recursive subdivision algorithm is employed here, which adaptively refines the sections of the median-dual that are cut by the interface. The node fluid volume is initialized to zero. At each stage of the recursion, the signed distance, $\phi_{p}$, for each vertex of each convex sub-polyhedron, $\vec{x}_{p}$, is calculated using the positional description of the surface. For each sub-polyhedron, if $\phi_{p}>0$ for all $\vec{x}_{p}$, the sub-polyhedron volume is added to the node fluid volume, whereas if $\phi_{p}<0$ for all $\vec{x}_{p}$, nothing is added to the node fluid volume, otherwise the interface intersects the sub-polyhedron. If the recursion level is less than the prescribed max level (taken as 4 in this work), each intersected sub-polyhedron is subdivided into sub-tetrahedra formed from the sub-polyhedron center, each face center, and the two nodes of each edge of each face (the number of generated sub-tetrahedra is equal to twice the number of edges in the convex subpolyhedron). If the recursion level is at the prescribed max level, approximate intersection points along each intersected edge are calculated using Eq. 13. The sub-polyhedron is truncated by a plane formed from three intersection nodes. The volume of the truncated sub-polyhedron is added to the node fluid volume. At the end of the recursive subdivision algorithm, the node fluid volume is divided by the volume of the node to get $F$. A stage of the recursion algorithm, which is less than the max number of prescribed recursion levels, is illustrated in the two-dimensional schematic of Fig. 11.

\subsection{Geometric operation time}

To assess the practicality of using the geometric tools described in Secs. 3.13.5 , we recorded the average times required to perform the operations on the non-convex median-dual node volumes from Fig. 10. Timings were collected using a single processor of a large memory node that utilizes dual 6-core X5650 $2.67 \mathrm{GHz}$ CPUs with $144 \mathrm{~GB}$ of RAM. Table 1 shows the average time to perform geometric operation on the non-convex polyhedra from Fig. 10 relative to the 


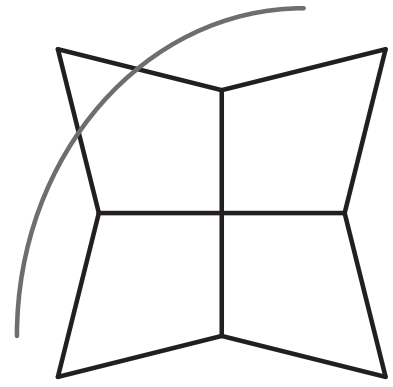

(a)

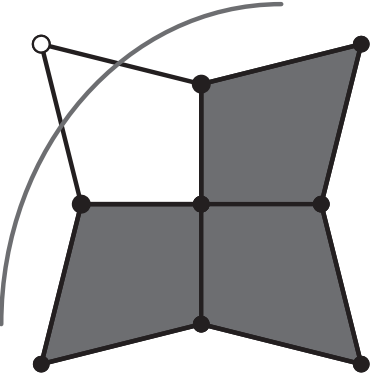

(b)

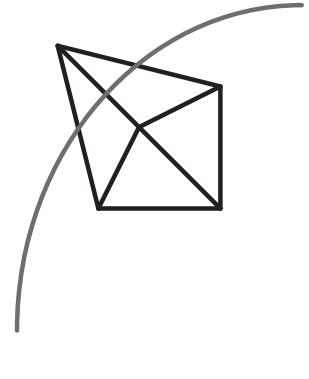

(c)

Figure 11: Stage of the recursive subdivision algorithm for initializing $F$ from a signed-distance function. An example of node volume with the interface crossing one convex sub-polygon is shown in (a). The convex sub-polyhedron is outlined by a solid black line and the interface is represented as a solid dark gray line in $(\mathrm{a}, \mathrm{b}, \mathrm{c})$. The computation of $\phi_{p}$ for the node volume, with $\phi_{p}>0$ shown by a filled black circle, and $\phi_{p}<0$ shown by an empty black circle, and the added fluid volume visualized with a dark gray region borded by a solid black line, is shown in (b). The subdivision of the sub-polygons into 4 sub-triangles is shown in (c).

time to truncate the joined non-convex polyhedron of Fig. 10b by a plane. The time to truncate the non-convex polyhedron by a plane was averaged over $10^{6}$ randomly positioned (inside the polyhedron) and oriented sample planes. The time to enforce the volume of the non-convex polyhedron by positioning a plane was averaged over $10^{6}$ randomly oriented sample planes and prescribed volumes (bound by the total polyhedron volume). The time to intersect the non-convex polyhedron with a repositioned and reoriented copy of itself was averaged over $10^{6}$ randomly positioned and oriented sample copies (that intersect with the polyhedron). The time to join the non-convex polyhedron was calculated only for unjoined non-convex polyhedron of Fig. 10a. As evidenced from Table 1, the times required to truncate the polyhedron by a plane, to enforce the volume of the polyhedron by repositioning a plane, and to intersect the polyhedron with a repositioned and reoriented copy of itself were all larger for the unjoined non-convex polyhedron than those of the joined non-convex polyhedron. For both the polyhedra in Figs. 10a and 10b, the intersection time was greater than the volume enforcement time; however, the times were much closer for 


\begin{tabular}{l|cccc} 
& Truncate & Enforce volume & Intersect & Join \\
\hline Fig. 10a & 9.2917 & 82.0131 & 221.2928 & 79.6084 \\
Fig. 10b & 1 & 9.4062 & 9.8940 & -
\end{tabular}

Table 1: The average time to perform geometric operation on the non-convex polyhedra from Fig. 10 relative to the time to truncate the joined non-convex polyhedron of Fig. $10 \mathrm{~b}$ by a plane.

the joined polyhedron than those of the unjoined polyhedron. Furthermore, the time required to intersect the unjoined polyhedron with a copy of itself was larger than the combined time to perform the join operation on both the polyhedron and its copy and to intersect them after. Therefore, joining each of the non-convex polyhedra prior to intersecting them saves both memory (fewer sub-polyhedra) and computation time.

\section{Surface normal and curvature tests}

Three primal mesh elements are used to test the EHF method, a hexahedron, a wedge, and a tetrahedron. The wedge and tetrahedron mesh elements' uniform edge lengths, $\Delta$, are prescribed such that their respective cell volumes match that of the hexahedral mesh of the same cell count. Specifically, $\Delta_{\text {wedge }}^{3} \approx 4 / \sqrt{3} \Delta_{\text {hexahedron }}^{3}$ and $\Delta_{\text {tetrahedron }}^{3} \approx 6 \sqrt{2} \Delta_{\text {hexahedron. Unfortunately, }}^{3}$ the edge constraints could only be approximately satisfied, so the wedge and tetrahedron elements are only approximately uniform in shape. Furthermore, the uniformity constraint on the edge length resulted in fewer cells in the wedgeand tetrahedron-packed primal meshes (see Fig. 12). Although the primal mesh elements are simple convex polyhedra, the node volumes of the median-dual mesh can be complicated in structure; e.g., the node volume of Fig. 10 was generated by the tetrahedral primal mesh in Fig. 12c.

Table 2 provides the total number of nodes $\left(N_{n o}\right)$, HF stencil units $\left(N_{s u}\right)$, and intersections of the HF stencil units with the node volumes $\left(N_{i n}\right)$ for the meshes in Fig. 12. The meshes were periodic, so $N_{s u}=135 \cdot N_{n o}$. For the 


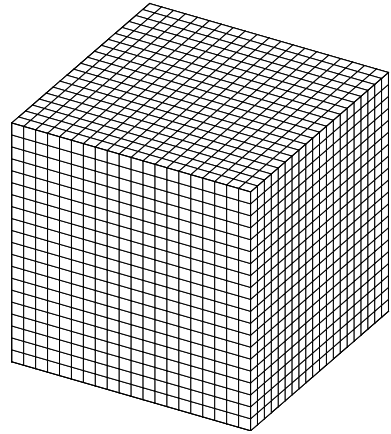

(a)

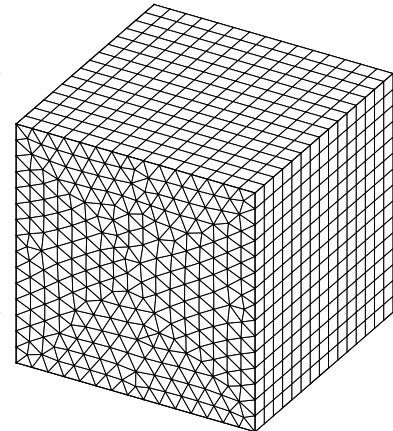

(b)

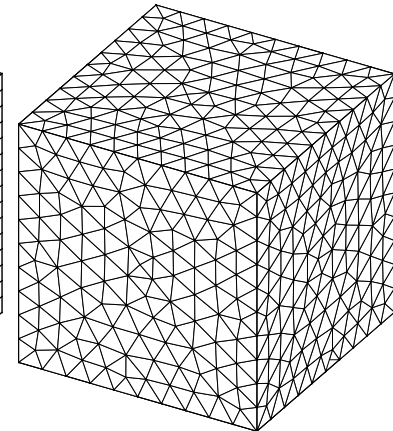

(c)

Figure 12: Example of primal meshes used in EHF tests of different topology. (a) A mesh composed of 8000 hexahedral elements. (b) A mesh of 7620 wedge elements is shown. (c) A mesh composed of 7302 tetrahedral elements is shown. The wedge and tetrahedron elements in (b) and (c) were created such that their volumes approximately matched a hexahedron element from (a).

\begin{tabular}{c|ccc} 
& $N_{n o}$ & $N_{s u}$ & $N_{i n}$ \\
\hline Hexahedron & 9261 & $1,250,235$ & $1,250,235$ \\
Wedge & 4560 & 615,600 & $2,731,048$ \\
Tetrahedron & 1589 & 214,515 & $2,132,429$
\end{tabular}

Table 2: The total number of nodes, HF stencil units, and intersections of the HF stencil units with the node volumes for the meshes in Fig. 12.

hexahedron element mesh (see Fig. 12a), $N_{i n}=N_{s u}$ because each HF stencil unit overlaps with a single node volume. For the wedge and tetrahedron element meshes (see Figs. 12b and 12c, respectively), each stencil-unit intersected with $N_{i n} / N_{s u}=4.436$ and 9.941 node volumes on average, respectively.

Three surfaces of known $\hat{n}$ and $\kappa$, embedded in a $8^{3}$ domain, are used to test the EHF technique: a sphere of radius 2, a cylinder of radius 2, and an ellipsoid of semi-principal axes lengths $2.3,2.0$, and 1.7. For all $\hat{n}$ and $\kappa$ tests, the error 
norms are defined as

$$
\begin{aligned}
& \mathcal{L}_{1}(\hat{n})=\frac{\sum_{p \epsilon \text { interface nodes }}\left|1-\hat{n}_{p}^{*} \cdot \hat{n}_{p}\right| V_{p}}{\sum_{p \epsilon \text { interface nodes }} V_{p}}, \\
& \mathcal{L}_{1}(\kappa)=\frac{\sum_{p \epsilon \text { interface nodes }}\left|\left(\kappa_{p}^{*}-\kappa_{p}\right) / \kappa_{p}^{*}\right| V_{p}}{\sum_{p \epsilon \text { interface nodes }} V_{p}},
\end{aligned}
$$

where $\hat{n}_{p}^{*}$ and $\hat{n}_{p}$ are, respectively, the true and calculated surface normals at node $p, \kappa_{p}^{*}$ and $\kappa_{p}$ are, respectively, the true and calculated surface curvatures at node $p$, and a node $p$ is considered an interface node if $0<F_{p}<1$. $\mathcal{L}_{\infty}(\hat{n})$ and $\mathcal{L}_{\infty}(\hat{\kappa})$ error norms were not used because they would bias towards the few isolated cells ( $<5$ for all tests), where less accurate auxiliary methods were used to calculate $\hat{n}$ and $\kappa$ (the PY and CV methods, respectively). To provide results that are representative of the possible situations with different relative alignments and locations of the PLIC interfaces and the mesh, the error norms in Eq. 16 are computed from 100 samples obtained by randomly positioning the surface.

\subsection{Volume fraction initialization}

Given the positional description of a surface, it is relatively easy to compute the signed distance, $\phi_{p}$, of an arbitrary position, $\vec{x}_{p}$, to the surface. For the three test-cases used to verify the EHF technique, $\phi_{p}$ is computed, respectively, as follows:

for a cylinder of radius $R$, centered at $\vec{x}_{o}$ along the $z$ axis,

$$
\phi_{p}=R-\sqrt{\left(x_{p}-x_{o}\right)^{2}+\left(y_{p}-y_{o}\right)^{2}}
$$

for a sphere of radius $R$, centered at $\vec{x}_{o}$,

$$
\phi_{p}=R-\sqrt{\left(x_{p}-x_{o}\right)^{2}+\left(y_{p}-y_{o}\right)^{2}+\left(z_{p}-z_{o}\right)^{2}}
$$

for an ellipsoid of semi-principal axis lengths $2 a, 2 b$, and $2 c$, centered at $\vec{x}_{o}$,

$$
\phi_{p} \approx \sqrt{a b c}\left(1-\sqrt{\left.\left(\left(x_{p}-x_{o}\right) / a\right)^{2}+\left(\left(y_{p}-y_{o}\right) / b\right)^{2}+\left(\left(z_{p}-z_{o}\right) / c\right)^{2}\right)}\right)
$$

where $\phi_{p}>0$ indicates that $\vec{x}_{p}$ is inside the surface. The correct computation of the $\phi_{p}$ for an ellipsoid would require a numerical procedure that enforced the 
eikonal equation, $\left\|\nabla \phi_{p}\right\|=1$, at every point, $\vec{x}_{p}$; however, Eq. 17 is accurate enough for initializing $F$ to second-order accuracy.

The true volume fraction for a node is not readily available for the test surfaces, which are non-planar, precluding the used of localized error norms. Following Lopez et al. [24], the error in the total volume, $\mathcal{L}_{1}(V)$, is used to assess the convergence of the $F$ initialization procedure. $\mathcal{L}_{1}(V)$, is calculated as

$$
\begin{aligned}
V & =\sum_{p \in \operatorname{\text {nodes}}} F_{p} V_{p} \\
\mathcal{L}_{1}(V) & =\left|V^{*}-V\right| / V^{*},
\end{aligned}
$$

where $V^{*}$ and $V$ are, respectively, the true and calculated volumes of fluid bounded by the surface. The geometric average number of nodes along a dimension, indicated by $N_{n o}^{1 / 3}$ in all figures, is the proper size parameter to check convergence against, because the median-dual mesh stores the collocated flow variables. Also, in all figures, first- and second-order convergence lines are plotted, respectively indicated as $\mathcal{O}\left(N_{n o}^{-1 / 3}\right)$ and $\mathcal{O}\left(N_{n o}^{-2 / 3}\right)$. The recursive procedure is shown to initialize $F$ to second order for different meshes and surfaces, as shown in Fig. 13.

\subsection{Impact of interpolation scheme}

Section 2.3 describes methods for interpolating $F_{p}$ from nodes $p$ that intersect HF stencil unit $u$ to $F_{u}$, the stencil-unit volume fraction. The methods are broken down into IVW and PLIC interpolation. Because the latter requires an estimate of $\hat{n}$ to perform the interpolation, the EHF method using IVW interpolation or an auxiliary method for estimating the $\hat{n}$ (e.g. the PY method) must be applied prior to performing the EHF method using PLIC interpolation. Further, $\hat{n}$ can be updated by the EHF method and the interpolation repeated. Methods are compared by checking the convergence of $\mathcal{L}_{1}(\hat{n})$ and $\mathcal{L}_{1}(\kappa)$ for a sphere of radius 2 in a domain of size $8^{3}$ over a series of tetrahedral primal meshes. The investigated methods are IVW interpolation, PLIC interpolation using $\hat{n}$ calculated from the PY method, IVW interpolation followed by PLIC 


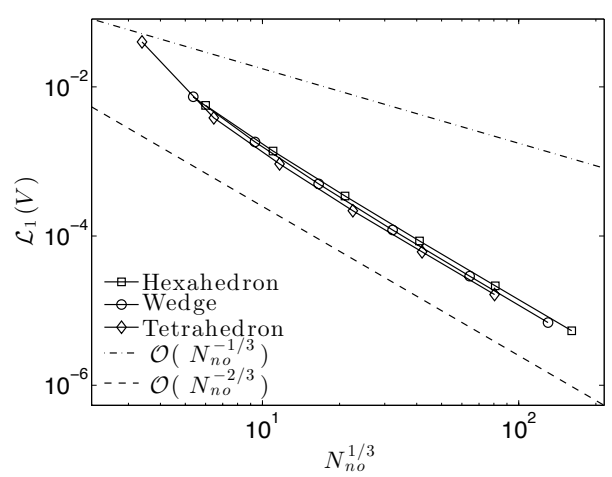

(a)

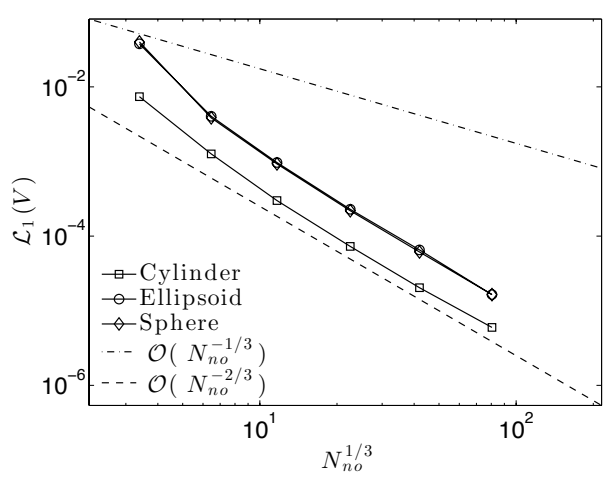

(b)

Figure 13: $\mathcal{L}_{1}(V)$ error from $F$ initialization. The $\mathcal{L}_{1}(V)$ error for a sphere of radius 2 embedded in a domain of size $8^{3}$ for hexahedron, wedge, tetrahedron primal meshes is shown in (a). The $\mathcal{L}_{1}(V)$ error for a sphere of radius 2 , a cylinder of radius 2 , and an ellipsoid of semiprincipal axis lengths $2.3,2.0$, and 1.7 , all embedded in a domain of size $8^{3}$, for tetrahedron primal mesh is shown in (b).

interpolation using $\hat{n}$ calculated from the EHF method, PLIC interpolation using $\hat{n}$ calculated from the PY method followed by PLIC interpolation using $\hat{n}$ calculated from the EHF method, PLIC interpolation using $\hat{n}$ calculated from the PY method followed by 9 iterations of PLIC interpolation using $\hat{n}$ calculated from the EHF method, and PLIC interpolation using the exact $\hat{n}$ for a sphere. The results of the study are shown in Fig. 14. From Fig. 14a, it is evident that the accuracy of $\hat{n}$ calculated using the EHF method is second-order convergent regardless of interpolation method or number of iterations; however, IVW interpolation was the least accurate, whereas PLIC interpolation using $\hat{n}$ calculated from the PY method followed by PLIC interpolation using $\hat{n}$ calculated from the EHF method was closest in accuracy to the PLIC interpolation using exact $\hat{n}$. The differences in the interpolation schemes are more apparent in $\mathcal{L}_{1}(\kappa)$, shown in Fig. 14b. Both IVW interpolation and PLIC interpolation using the PY method begin to diverge for large $N_{n o}$. The methods using at least two iterations continue to converge; however, PLIC interpolation for the first iteration was more accurate than IVW interpolation. Following the first iteration of 


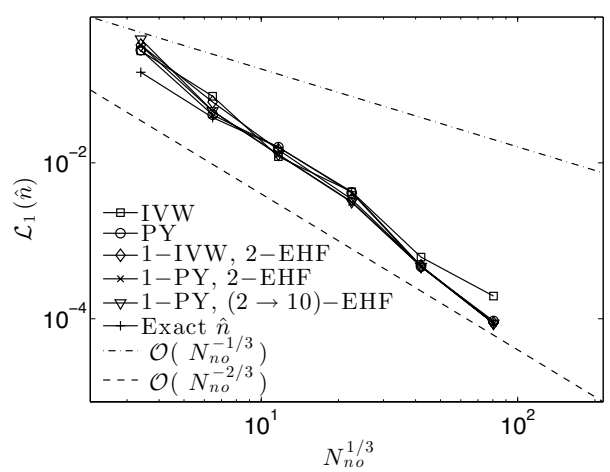

(a)

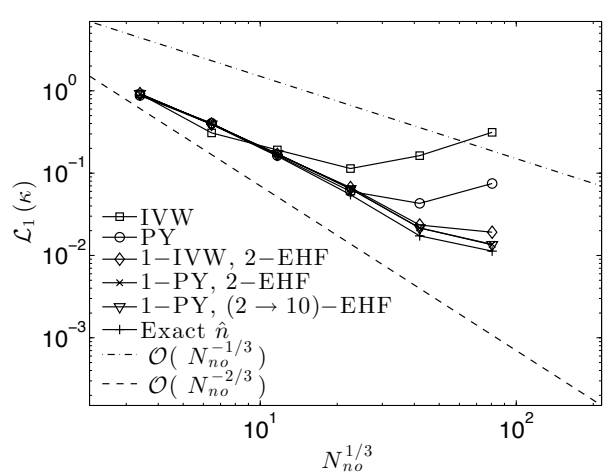

(b)

Figure 14: $\mathcal{L}_{1}$ Errors in $\hat{n}(\mathrm{a})$ and $\kappa(\mathrm{b})$ for a sphere of radius 2 in a domain of size $8^{3}$ using different interpolation schemes on a tetrahedral primal mesh. IVW indicates IVW interpolation. PY indicates PLIC interpolation using $\hat{n}$ calculated from the PY method. $1-\mathrm{IVW}$, $2-$ EHF indicates IVW interpolation followed by PLIC interpolation using $\hat{n}$ calculated from the EHF technique. 1-PY, 2-EHF indicates PLIC interpolation using $\hat{n}$ calculated from the PY method followed by PLIC interpolation using $\hat{n}$ calculated from the EHF technique. $1-\mathrm{PY},(2 \rightarrow 10)$-EHF indicates PLIC interpolation using $\hat{n}$ calculated from the PY method followed by 9 iterations of PLIC interpolation. Exact $\hat{n}$ indicates PLIC interpolation using the exact $\hat{n}$ for the sphere.

PLIC interpolation using the PY method, $\mathcal{L}_{1}(\kappa)$ is insensitive to the number of subsequent iterations of PLIC interpolation using $\hat{n}$ calculated from the EHF method, showing a monotonically decreasing error that is slightly larger than that of interpolation using exact $\hat{n}$. Each iteration requires volume enforcement of $\hat{n}$ to reconstruct the planar interface and an expensive interpolation of $F$ from the median-dual mesh and to the embedded HF stencils, which encompasses the bulk of the calculation time. In light of the insensitivity of $\mathcal{L}_{1}(\kappa)$ to the number of iterations of PLIC interpolation after two, we implemented PLIC interpolation using $\hat{n}$ calculated from the PY method followed by PLIC interpolation using $\hat{n}$ calculated from the EHF method for the remainder of the tests. 


\subsection{Impact of smoothing parameter}

Note that in Fig. 14b, even for PLIC interpolation using exact $\hat{n}$, the convergence rate in $\mathcal{L}_{1}(\kappa)$ decays for large $N_{n o}$. As a heuristic means to maintain the accuracy for large $N_{n o}$, the calculations of $\hat{n}$ and $\kappa$ using Eq. 6 have an implicit dependence on $\gamma$ from the smoothed finite difference formulas in Eq. 7 . The parameter was proposed as a heuristic means to increase the accuracy of $\kappa$ by addressing $H$ errors due to misalignment of the HF direction and $\hat{n}$ [24]. $\gamma$ was prescribed as

$$
\gamma=\left\{\begin{array}{ll}
0.2 & \text { if } \theta \geq \theta_{\text {crit }} \\
0 & \text { otherwise }
\end{array},\right.
$$

where $\theta=\operatorname{acos}\left(n_{z}\right)$ ( $z$ is assumed to be the HF direction), and $\theta_{\text {crit }}=0.8$.

Errors in $H, e$, are amplified by numerical differentiation. Specifically, the errors in $H_{x}$ and $H_{x x}$ using second-order finite differences are

$$
\begin{aligned}
E\left(H_{x}\right) & =\left(e_{1}-e_{-1}\right) /(2 \Delta x)+\mathcal{O}\left(\Delta x^{2}\right) \approx e / \Delta x+\mathcal{O}\left(\Delta x^{2}\right), \\
E\left(H_{x x}\right) & =\left(e_{1}-2 e_{0}+e_{-1}\right) / \Delta x^{2}+\mathcal{O}\left(\Delta x^{2}\right) \approx 4 e / \Delta x^{2}+\mathcal{O}\left(\Delta x^{2}\right),
\end{aligned}
$$

so for small $\Delta x$, errors in $H_{x}$ and $H_{x x}$ are dominated by errors in $H$. From Eqs. 6 and $7, e$ is approximately amplified by $\mathcal{O}(1 / \Delta x)$ in the calculation of $\hat{n}$ and is approximately amplified by $\mathcal{O}\left(1 / \Delta x^{2}\right)$ in the calculation of $\kappa$. The smoothing procedure attempts to minimize the impact of $e$ on $\hat{n}$ and $\kappa$. For uniform cartesian meshes, $e$ is mostly due to misalignment of the HF direction from $\hat{n}$, so smoothing based on $\theta$ is optimal [24]. In the EHF method on unstructured grids, $e$ is induced from misalignment of the HF direction with $\hat{n}$ and from the interpolation procedure. As a heuristic means to address this added source of error, which is independent of the misalignment of the HF direction with $\hat{n}$, we compare the accuracy of various constant values of $\gamma$ to that of Eq. 19.

The results of the study are shown in Fig. 15. From Fig. 15a, it is evident that $\mathcal{L}_{1}(\hat{n})$ is insensitive to the choice in $\gamma$; this insensitivity is due to the smaller amplification of $e$ in $\hat{n}, \mathcal{O}(1 / \Delta x)$. Although it is visually difficult to discern, $\gamma \neq 0$ slightly increases the error in $\hat{n}$, so we chose not to smooth the numerical derivates in the computation of $\hat{n}$ from the EHF method. The $\hat{n}$ from 


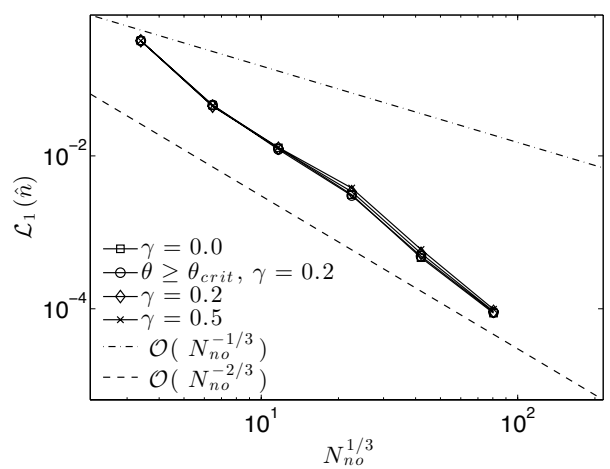

(a)

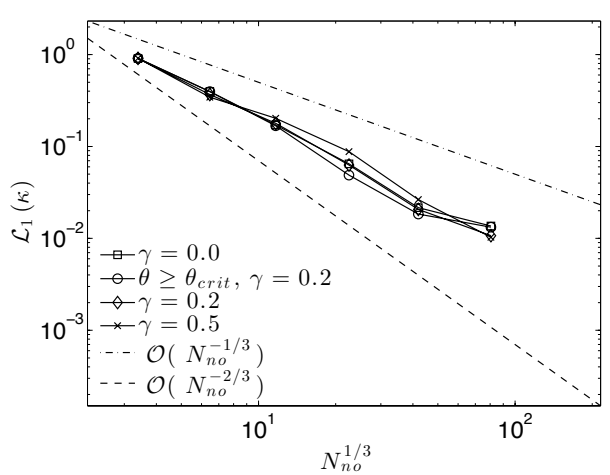

(b)

Figure 15: $\mathcal{L}_{1}$ errors in $\hat{n}(\mathrm{a})$ and $\kappa(\mathrm{b})$ for a sphere of radius 2 in a domain of size $8^{3}$ using different finite-difference smoothers on a tetrahedral primal mesh.

the EHF method is used for PLIC interpolation during the second iteration, so errors in $\hat{n}$ impact the accuracy of $\kappa$. The results in Fig. 15b are constructed without smoothing the derivatives in the computation of $\hat{n}$. From Fig. $15 \mathrm{~b}$ it is evident that $\mathcal{L}_{1}(\kappa)$ is sensitive to the choice of $\gamma$, due to the larger amplification of $e$ in $\kappa, \mathcal{O}\left(1 / \Delta x^{2}\right)$. Smoothing based on Eq. 19, where $\gamma=0.2$ if $\theta \geq$ $\theta_{\text {crit }}$, reduced $\mathcal{L}_{1}(\kappa)$ for all $N_{\text {no }}$; however, the impact diminishes at larger $N_{n o}$, suggesting that errors in $H$ due to misalignment of the HF direction and $\hat{n}$ are not dominant at the highest resolutions. Smoothing with a constant $\gamma$ decreases $\mathcal{L}_{1}(\kappa)$ for the largest $N_{n o}$; however, for $\gamma=0.5, \mathcal{L}_{1}(\kappa)$ increases for smaller $N_{n o}$. $\mathcal{L}_{1}(\kappa)$ for a constant $\gamma=0.2$ approximately followed that for $\gamma=0.0$, only decreasing the error for the largest $N_{n o}$. Because a constant $\gamma=0.2$ aided in the convergence of the EHF method for a larger range of $N_{n o}$, we chose to use it as the smoother for the remainder of the tests (with $\gamma=0.0$ for the calculation of $\hat{n}$ ). A more intelligent smoothing procedure that uses Eq. 19 for smaller $N_{n o}$ and a larger constant $\gamma$ for larger $N_{n o}$ could be used; however, the transition $N_{n o}$ is dependent on the primal mesh, so we did not pursue this approach. 


\subsection{Impact of primal mesh}

The EHF technique reduces to standard HF for a uniform hexahedral primal mesh. The median dual of a uniform hexahedral primal mesh is hexahedral (and uniform internally), so an HF stencil-unit bounding box aligns with a single node volume. Because an HF stencil-unit and a node volume are equivalent, the $F$ 's match and no interpolation procedure is necessary. We inspect the error induced by embedding stencils and interpolating by comparing $\mathcal{L}_{1}(\hat{n})$ and $\mathcal{L}_{1}(\kappa)$ for a wedge and tetrahedral primal mesh to those of the hexahedral primal mesh. The results of the study are shown in Fig. 16. As shown in Fig. 16a, $\mathcal{L}_{1}(\hat{n})$ is asymptotically second-order accurate for all primal meshes; however, there is a loss in precision for the wedge and tetrahedral primal meshes, with the latter being the least accurate. The lower accuracy at small $N_{n o}$ on the hexahedral primal mesh can be attributed to the utilized definition of the HF (see Sec. 2.1). The convergence behavior of $\mathcal{L}_{1}(\kappa)$ in Fig. $16 \mathrm{~b}$ is impacted more by the primal mesh. $\mathcal{L}_{1}(\kappa)$ converges at a slightly lower rate for the wedge and tetrahedral primal meshes than for the hexahedral primal mesh. Furthermore, the precision of $\kappa$ on the wedge primal meshes begins to saturate at large $N_{n o}$, suggesting that the error in $\kappa$ from truncation of the Taylor series begins to balance those from $e$, which are amplified by $\mathcal{O}\left(1 / \Delta x^{2}\right)$.

In the absence of misalignment of the HF direction with $\hat{n}$, the errors in $H$ are solely based on the errors in $F$ in the HF stencil due to interpolation from the unstructured mesh. As described in the Sec. 4.2, the chosen EHF method requires two iterations, PLIC interpolation using $\hat{n}$ calculated from the PY method followed by PLIC interpolation using $\hat{n}$ calculated from the first iteration of the EHF method. In this approach, the accuracy of the $F$ interpolation of the second iteration is dictated by the accuracy of $\hat{n}$ calculated during the first iteration of the EHF method (PLIC interpolation using $\hat{n}$ calculated from the PY method), which was shown to be approximately second-order accurate in Fig. 14a. With a second-order estimate of $\hat{n}$, the errors in the stencil-unit volume fraction are second-order accurate. In the absence of multiple interfaces in the stencil, the errors of $H$ are a simple sum of the errors in the volume fraction 


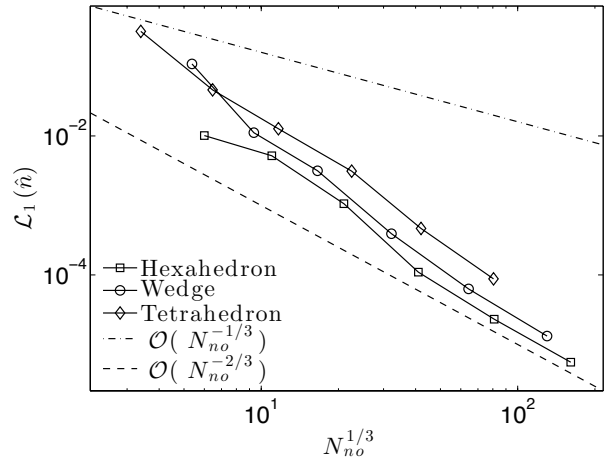

(a)

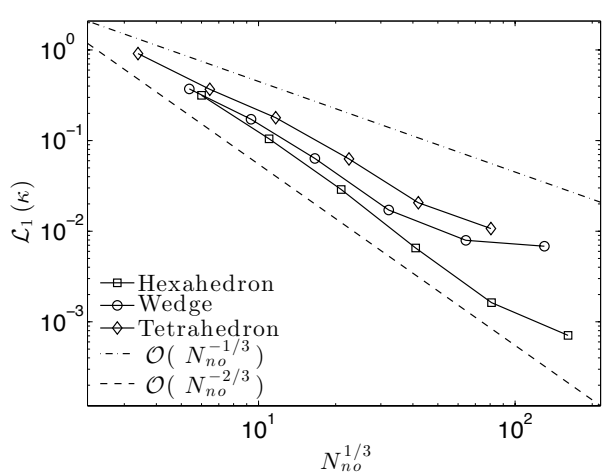

(b)

Figure 16: $\mathcal{L}_{1}$ errors in $\hat{n}(\mathrm{a})$ and $\kappa(\mathrm{b})$ for a sphere of radius 2 in a domain of size $8^{3}$ on different primal meshes.

over the column and are second-order accurate as well, $e \approx c \Delta x^{2}$. Substituting this definition of $e$ into Eq. 20 provides estimates for errors in derivates in $H$,

$$
\begin{aligned}
E\left(H_{x}\right) & \approx c \Delta x+\mathcal{O}\left(\Delta x^{2}\right), \\
E\left(H_{x x}\right) & \approx 4 c+\mathcal{O}\left(\Delta x^{2}\right),
\end{aligned}
$$

where $c$ is small. The actual magnitude of $c$ depends on the accuracy of $\hat{n}$ used to perform the PLIC interpolation (e.g. the PLIC interpolations using at least two iterations in Fig. 16b), the primal mesh (e.g. the tetrahedral and wedge primal meshes in Fig. 16b), and the construction of $H$ from Eqs. 3-7, making it difficult to estimate. The important point is that $E\left(H_{x}\right)$ and $E\left(H_{x x}\right)$ do not grow with $N_{n o}$, so from Eq. 6 we know that $\mathcal{L}_{1}(\hat{n})$ and $\mathcal{L}_{1}(\kappa)$ monotonically decrease with $N_{n o}$. A similar decay in convergence with mesh refinement was demonstrated by the $\hat{n}$-aligned HF method [26].

\subsection{Comparison with other methods}

The calculation of $\hat{n}$ and $\kappa$ in PLIC-VOF methods on three-dimensional unstructured non-convex polyhedral meshes is underdeveloped. The PY method, because of its simplicity, remains a popular technique to estimate $\hat{n}$, while estimations of $\kappa$ are traditionally performed with the CV or the RDF methods. In 
the former method, the $F$ data is convolved with a kernel function (taken from Cummins et al. [15]). In the latter method, a signed-distance field is constructed from the PLIC plane, requiring an estimate of $\hat{n}$ (assumed to come from the PY method here). To have another method to estimate $\hat{n}$, we use gradients of the RDF to update the estimate of $\hat{n}$ calculated from the PY method. The unstructured gradient, divergence, and Laplacian operators of our collocated flow solver [6] are used for differentiating variables on the median dual. The RDF is generated using the method described by Cummins et al. [15], where the power of the weighting function is taken as 10. To compare the EHF method to a proven second-order accurate method, we also calculate $\hat{n}$ using LVIRA. LVIRA implements a two-dimensional least-squares minimization procedure on each dual volume, where each iterate requires volume enforcement as well as volume truncation for each node in the local dual-volume neighborhood. The minimization of the non-linear function of two variables is performed using Powell's method in conjunction with Brent's method. Figures 17-19 plot $\mathcal{L}_{1}$ errors in $\hat{n}$, computed using the EHF, PY, RDF methods and LVIRA, and plot $\mathcal{L}_{1}$ errors in $\kappa$, computed using the EHF, CV, and RDF methods, for hexahedral, wedge, and tetrahedral primal meshes. Note that, in these figures, we did not include convergence plots in $\kappa$ using its definition from Eq. 2 combined with any of the methods used to calculate $\hat{n}$ (e.g. LVIRA), as this would require taking the divergence of a discontinuous field on an unstructured mesh.

Figure 17 plots $\mathcal{L}_{1}$ errors in $\hat{n}$ and $\kappa$ for a sphere of radius 2 in a domain of size $8^{3}$. The results for $\hat{n}$ are shown in the left column of the figure. The PY method fails to produce even first-order convergence. The RDF method fails to converge for the hexahedral primal mesh, but provides greater than first-order accurate results for the wedge and tetrahedral primal meshes at low-to-moderate $N_{n o}$. The RDF method is shown to be more accurate than the PY method for all meshes. The EHF method produces normals that are asymptotically secondorder accurate on the hexahedral primal mesh; however, the convergence rate is lower for small $N_{n o}$, suggesting that the surface is under-resolved by the standard HF method [19]. For the tetrahedral and wedge primal meshes, the EHF 


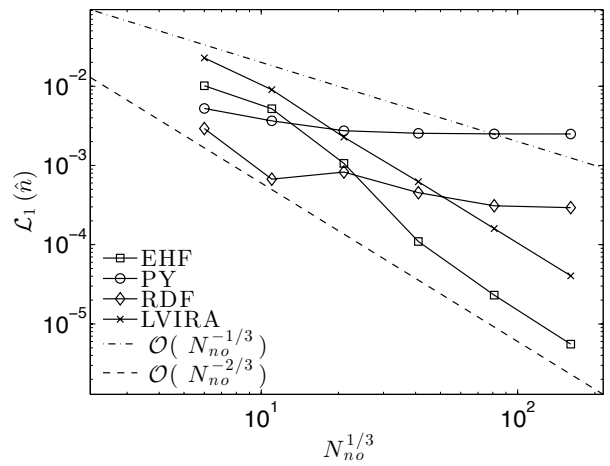

(a)

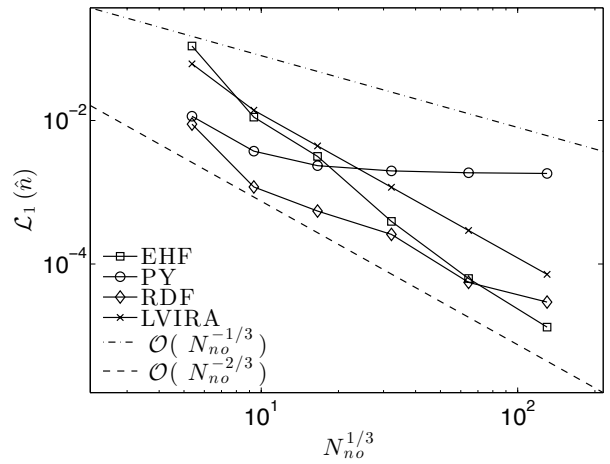

(c)

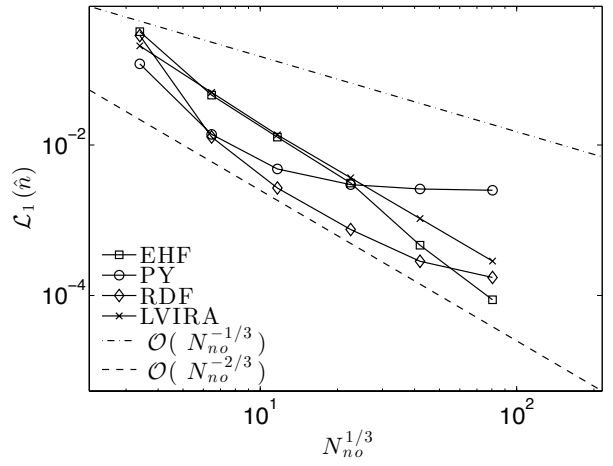

(e)

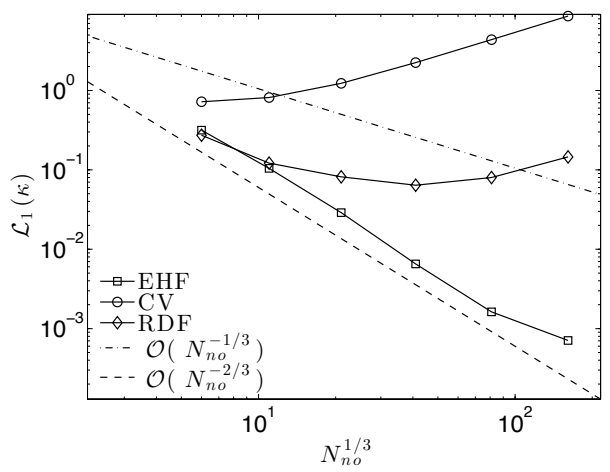

(b)

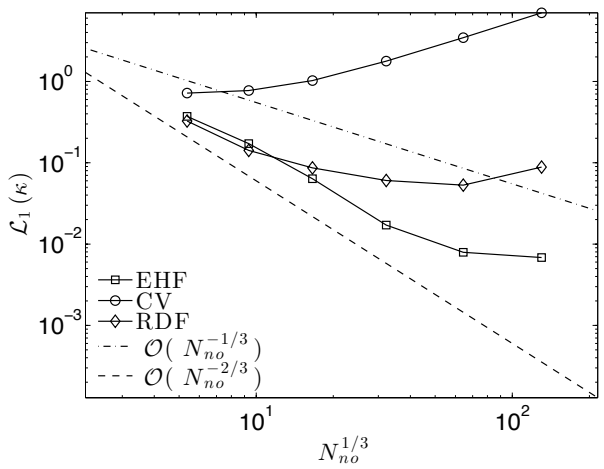

(d)

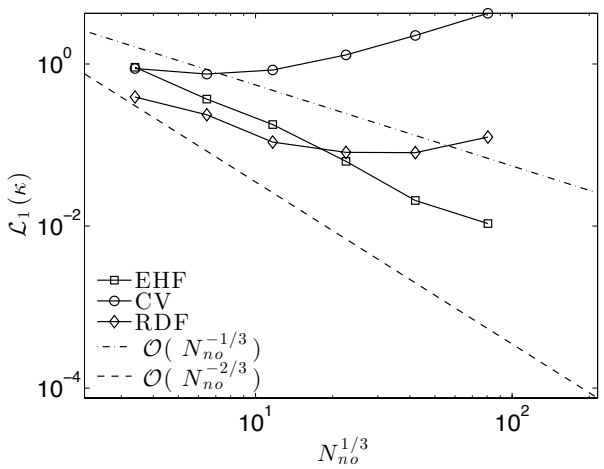

(f)

Figure 17: $\mathcal{L}_{1}$ errors in $\hat{n}(\mathrm{a}, \mathrm{c}, \mathrm{e})$ and $\kappa(\mathrm{b}, \mathrm{d}, \mathrm{f})$ for a sphere of radius 2 in a domain of size $8^{3}$ on hexahedral primal meshes $(\mathrm{a}, \mathrm{b})$, wedge primal meshes $(\mathrm{c}, \mathrm{d})$, and a tetrahedral primal meshes $(\mathrm{e}, \mathrm{f})$. 
method converges with at least second-order accuracy for the entire range of $N_{n o}$. For low resolutions, the accuracy of the EHF method is lower than that of the PY and RDF methods. LVIRA converges to second order; however, it is less precise than the EHF method for hexahedral meshes at all resolutions and for wedge and tetrahedral meshes at all but the lowest resolutions. From the tetrahedral and primal mesh EHF test cases, it is evident that the interpolation errors, amplified by $\mathcal{O}(1 / \Delta x)$, are not leading order for the range this range of $N_{n o}$. The results of the $\kappa$ calculation are shown in the right column of the figure. The CV method produces errors that increase with resolution. The RDF method is more accurate than the CV method; however, at moderate-tohigh $N_{n o}$, its numerical errors increase as well. The growth in $\mathcal{L}_{1}(\kappa)$ for the $\mathrm{CV}$ and the RDF methods has been reported by previous researchers for twodimensional structured [15] and unstructured [21] meshes. The EHF method produces monotonically decreasing errors, showing approximately second-order convergence on hexahedral primal meshes and greater than first-order convergence on tetrahedral primal meshes for all but the largest $N_{n o}$. For the wedge primal mesh, the precision of the EHF method saturates at the highest resolutions, reducing the convergence rate; this suggests that the interpolation errors, amplified by $\mathcal{O}\left(1 / \Delta x^{2}\right)$, have become leading order. The EHF method produces more precise curvatures than those of the CV method for all but the smallest $N_{n o}$ on the tetrahedral primal mesh and those of RDF method at moderateto-high resolutions. While the $\mathrm{CV}$ and $\mathrm{RDF}$ techniques employ second-order discretizations, the second derivates of these functions do not remain bounded under mesh refinement, so their associated estimates of $\kappa$ lose accuracy at higher resolutions [15].

Figure 18 shows $\mathcal{L}_{1}$ errors in $\hat{n}$ and $\kappa$ for a cylinder of radius 2 in a domain of size $8^{3}$. The results of the $\hat{n}$ calculation are shown in the left column of the figure. Similar to the sphere test, the errors in the PY method only decrease over the lowest resolutions. The EHF method again converges with at least second-order accuracy for the tetrahedral and wedge primal meshes and with asymptotic second-order accuracy for the hexahedral primal mesh. The RDF 


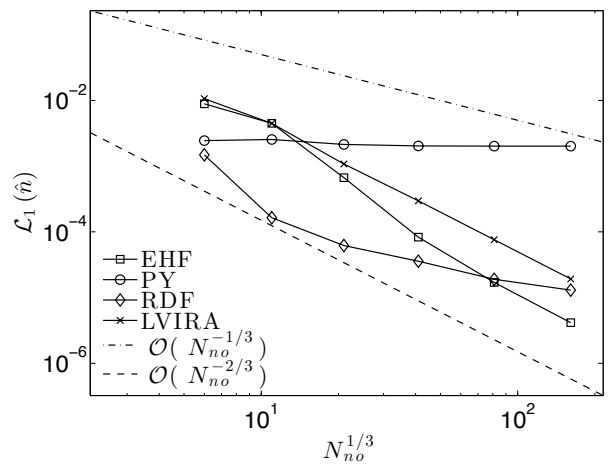

(a)

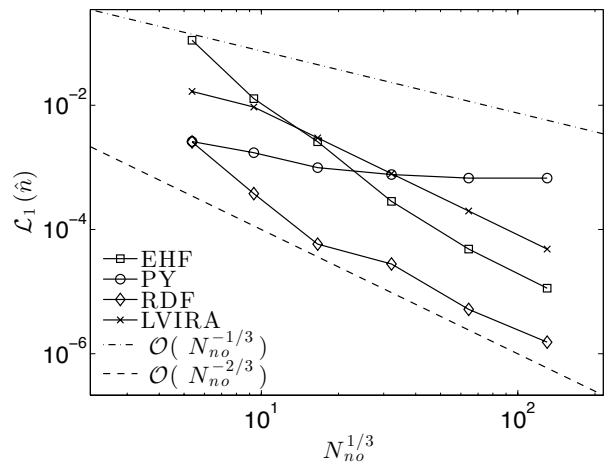

(c)

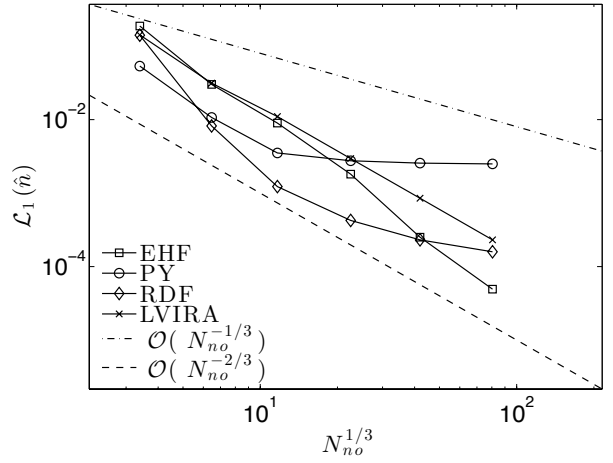

(e)

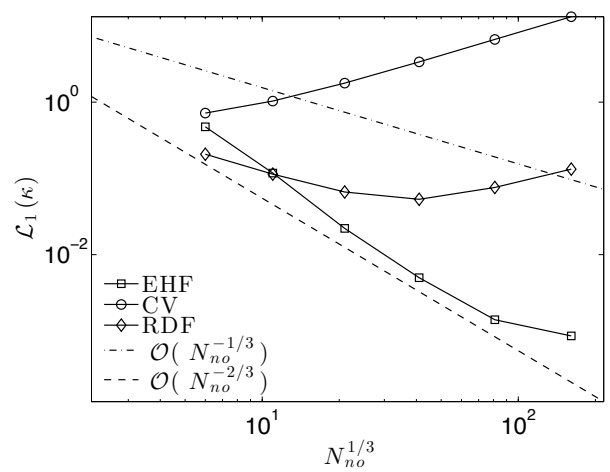

(b)

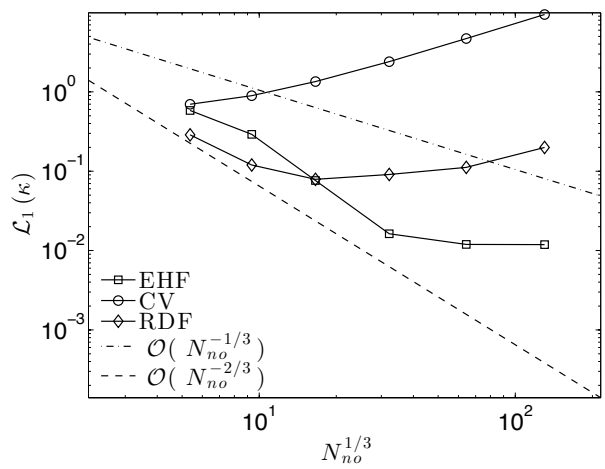

(d)

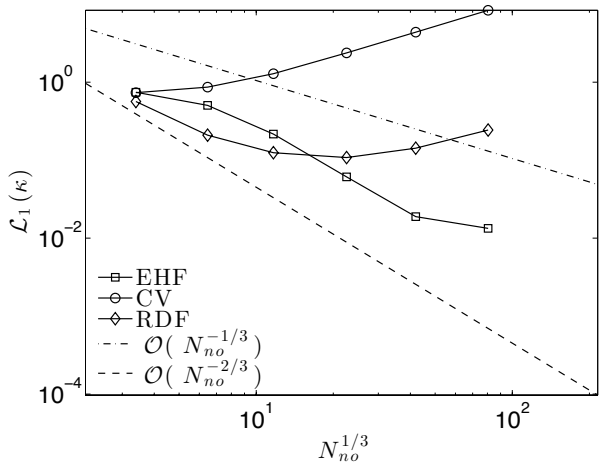

(f)

Figure 18: $\mathcal{L}_{1}$ errors in $\hat{n}(\mathrm{a}, \mathrm{c}, \mathrm{e})$ and $\kappa(\mathrm{b}, \mathrm{d}, \mathrm{f})$ for a cylinder of radius 2 in a domain of size $8^{3}$ on hexahedral primal meshes $(\mathrm{a}, \mathrm{b})$, wedge primal meshes $(\mathrm{c}, \mathrm{d})$, and a tetrahedral primal meshes $(\mathrm{e}, \mathrm{f})$. 
method is more accurate than the PY method and is even more accurate than the EHF method for the wedge primal mesh, showing approximately secondorder convergence. For the hexahedral and tetrahedral meshes, the RDF method converges over small $N_{n o}$. LVIRA converges to second-order; however, with less precision than the EHF technique for all but the lowest $N_{n o}$. The results of the $\kappa$ calculation for the cylinder are shown at the right side of the figure. Just as with the sphere test case, the CV method's errors increase with greater resolution and the RDF method's errors decrease at lower resolutions and increase at higher resolutions. The EHF method's errors decrease with resolution and converge with rates slightly higher than that of the sphere for the same mesh at moderate resolution; however, the precision saturates for the wedge primal mesh at a smaller $N_{n o}$ than that of the sphere and the convergence rates for the hexahedral and tetrahedral primal meshes are lower than that of the sphere for the same mesh at the highest $N_{n o}$. The EHF method is more precise than the CV method for all $N_{n o}$ and the RDF method for moderate-to-high $N_{n o}$.

Figure 19 plots $\mathcal{L}_{1}$ errors in $\hat{n}$ and $\kappa$ for an ellipsoid of semi-principal axes lengths $2.3,2.0$, and 1.7 in a domain of size $8^{3}$. The results of the $\hat{n}$ calculation are shown in the left column of the figure. Similar to the sphere and cylinder tests, the PY method has less than unity convergence. The EHF method is at least second-order convergent for all meshes at all resolutions. The RDF method is more accurate than the PY method and shows greater than first-order convergence on the wedge and tetrahedral primal meshes for low-to-moderate $N_{n o}$; however, the technique suffers in convergence on the hexahedral primalmesh. LVIRA, as compared to the EHF technique, shows similar convergence and accuracy trends as it does for the cylinder. $\kappa$ results are displayed in the right column of the figure. Just as with the sphere and cylinder tests, the CV method's errors increase with greater resolution and the RDF method's errors decrease at lower resolutions and increase at higher resolutions. The EHF method's errors decrease with greater resolution, but the errors converge with slightly slower rates than those of the sphere and cylinder for the same mesh at low-to-moderate $N_{n o}$. Similar to the sphere test, the EHF method 


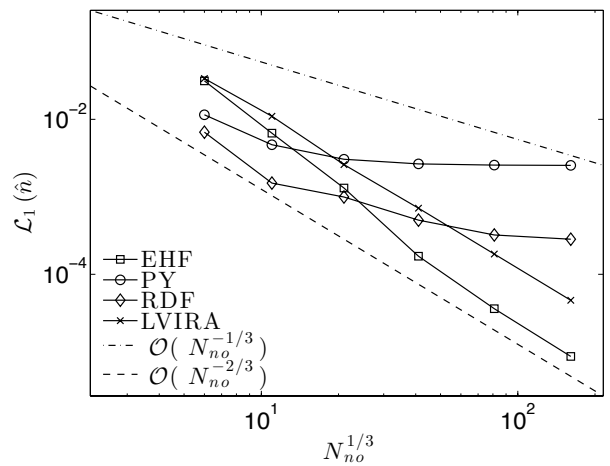

(a)

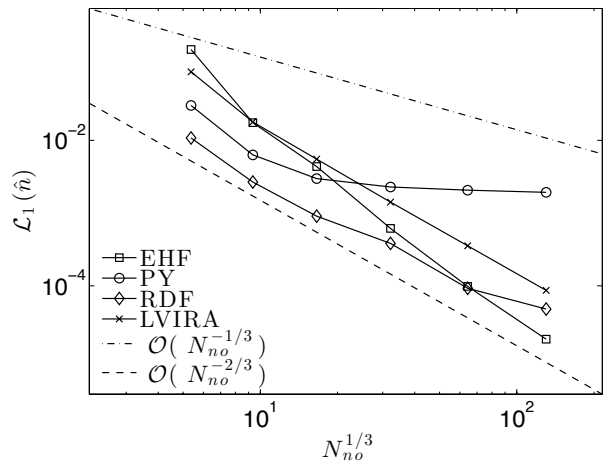

(c)

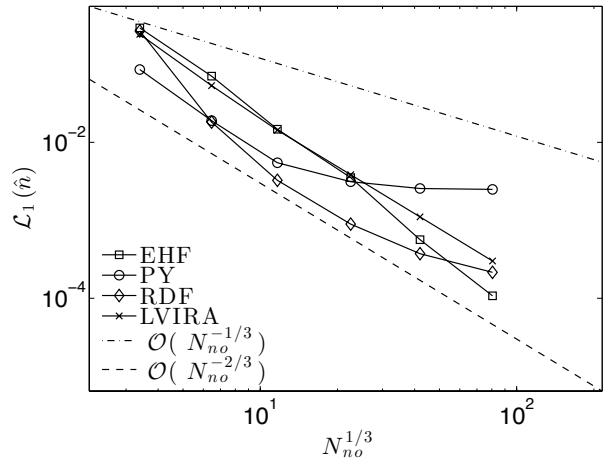

(e)

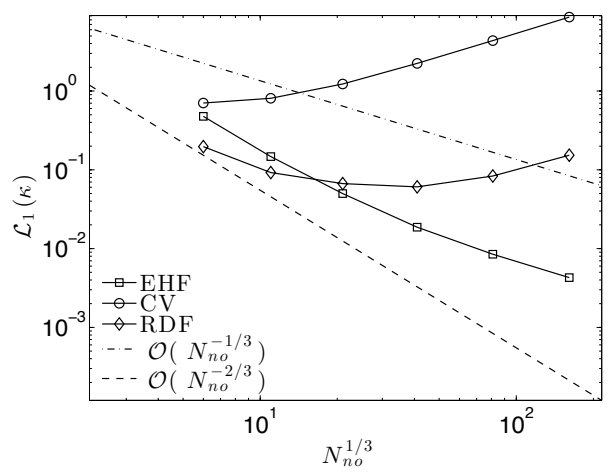

(b)

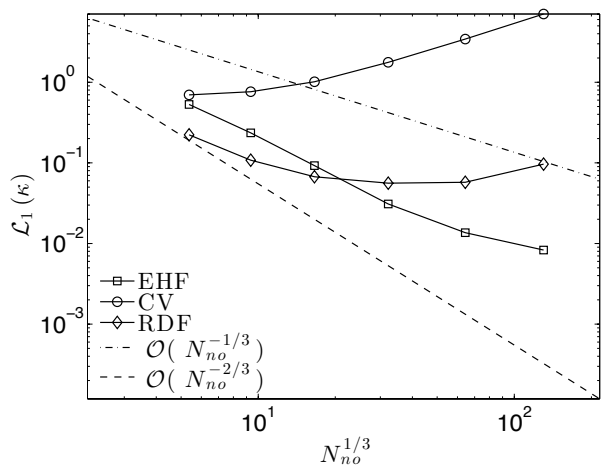

(d)

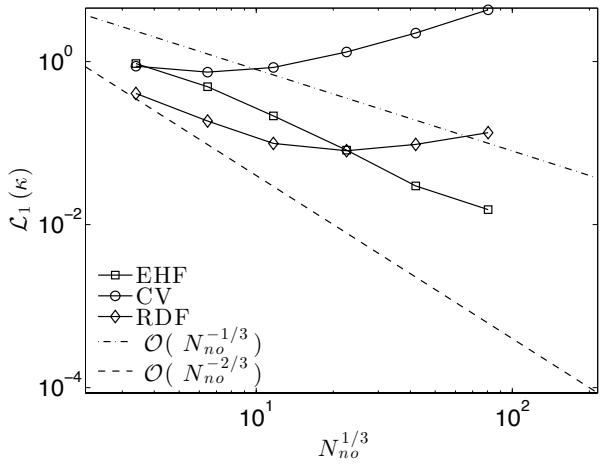

(f)

Figure 19: $\mathcal{L}_{1}$ errors in $\hat{n}(\mathrm{a}, \mathrm{c}, \mathrm{e})$ and $\kappa(\mathrm{b}, \mathrm{d}, \mathrm{f})$ for an ellipsoid of semi-principal axes lengths $2.3,2.0$, and 1.7 in a domain of size $8^{3}$ on hexahedral primal meshes (a,b), wedge primal meshes $(c, d)$, and a tetrahedral primal meshes $(e, f)$. 
produces more precise curvatures than those of the CV method for all but the smallest $N_{n o}$ on the tetrahedral primal mesh and those of RDF method at moderate-to-high resolutions.

To gauge the cost of the EHF method, we compared the times to calculate $\hat{n}$ and $\kappa$ with the EHF method to that of the time to calculate $\hat{n}$ using LVIRA, the only other second-order convergent method (in $\hat{n}$ ). To avoid costs associated to the parallel implementation differences, timings were collected using a single processor of a large memory node that utilizes dual 6-core X5650 2.67 GHz CPUs with 144 GB of RAM. Timings for both the EHF method and LVIRA depend on the initial estimate of $\hat{n}$ (calculated from the PY method), so times were averaged across 100 samples obtained from randomly positioning the surface in the primal mesh. The ratio of the times were found to not depend on the number of nodes of a given element type in the primal mesh, so the ratio for all the mesh sizes were averaged to give a single relative cost estimate for a given primal mesh element (hexahedron, wedge, tetrahedron) and surface (sphere, cylinder, ellipsoid). Table 3 shows the average ratio of the time to calculate $\hat{n}$ using LVIRA over the time to calculate $\hat{n}$ and $\kappa$ with the EHF method, $T_{\mathrm{LVIRA}} / T_{\mathrm{EHF}}$. $T_{\mathrm{LVIRA}} / T_{\mathrm{EHF}}$ is largest for tetrahedron primal meshes and second largest for wedge primal mesh; this is due to the increased number of sub-polyhedra in the

convex decomposition of the median-dual volume. $T_{\mathrm{LVIRA}} / T_{\mathrm{EHF}}$ is larger for the three-dimensional surfaces, sphere and ellipsoid, than for the two-dimensional cylinder; this comes from the effective removal of one of the dimensions from the least-squares minimization procedure in LVIRA. Across the tests, LVIRA requires $\mathcal{O}(10-100)$ times more wall-clock time than the EHF method, which justifies the removal of LVIRA as a candidate to estimate $\hat{n}$ in the geometric interpolation procedure of the EHF method.

\section{Conclusions}

We developed an accurate method to calculate interface normals and curvatures on a three-dimensional unstructured mesh composed of non-convex poly- 


\begin{tabular}{c|ccc} 
& Sphere & Cylinder & Ellipsoid \\
\hline Hexahedron & 25.47 & 18.05 & 17.71 \\
Wedge & 58.41 & 58.76 & 53.06 \\
Tetrahedron & 121.8 & 80.66 & 104.1
\end{tabular}

Table 3: The average ratio of the time to calculate $\hat{n}$ using LVIRA over the time to calculate $\hat{n}$ and $\kappa$ with the EHF method, $T_{\mathrm{LVIRA}} / T_{\mathrm{EHF}}$.

hedra. The method embeds traditional height-function stencils in the unstructured mesh and geometrically interpolates the volume fraction information from the mesh to the stencil. Two methods for interpolating the volume fraction information were developed, a first-order accurate intersection volume-weighted method and a second-order accurate PLIC method.

To perform both PLIC-VOF on non-convex polyhedral meshes and the intersections necessary for interpolation methods, we developed a series of geometric tools that utilize the convex-decomposition description of a non-convex polyhedron: truncation of a polyhedron by a plane, volume enforcement of a polyhedron by a plane, intersection of two polyhedra, joining of convex polyhedra, and volume fraction initialization by a signed-distance function.

To validate the embedded height-function method, the interface normal and curvature for a sphere, a cylinder, and an ellipsoid were calculated on the median duals of hexahedral, wedge, and tetrahedral primal meshes. The tests demonstrated that the embedded height-function method calculated interface normals with approximately second-order accuracy and calculated curvature with monotonically decreasing errors. At moderate-to-high resolutions, the method was nominally more accurate than the methods currently in use. For the calculation of the interface normal, the embedded height-function technique was more accurate and far less costly than the least-squares volume-of-fluid interface reconstruction algorithm, a second-order accurate method. To have an additional method to compare with, we proposed using the reconstructed distance function, traditionally used for curvature, to estimate the normal. This method 
required an approximation of the normal to reconstruct a planar interface from which the signed distances were constructed. The reconstructed distance function provided a more accurate alternative to the Parker-Young's method; however, its convergence behavior was surface and mesh dependent. The embedded height-function technique was the only method that had monotonically decreasing errors in the calculation of curvature.

The convergence of the embedded height-function method in curvature began to saturate on wedge and tetrahedral meshes of the highest resolution. The reduction of the convergence rate at high resolutions owes to errors in the computed heights, which are mainly attributed to the interpolation of the volume fraction data from the unstructured mesh to the embedded height-function stencils. As a first attempt at reducing the impact of the errors in the computed heights on the curvature calculation, we smoothed the finite-difference operators; however, the convergence rate still decreased at the highest resolutions. A more thorough investigation of the errors induced by geometric interpolation

of discontinuous data may yield insight in increasing the range over which the method converges.

\section{Ackowledgements}

This work was supported by the Department of Energy Computational Science Graduate Fellowship under grant number DE-FG02-97ER25308 and by the Stanford Graduate Fellowship.

\section{References}

[1] R. Scardovelli, S. Zaleski, Direct Numerical Simulation of free-surface and interfacial flow, Annu. Rev. Fluid Mech. 31 (1999) 567-603.

[2] G. Tryggvason, R. Scardovelli, S. Zaleski, Direct Numerical Simulations of Gas-Liquid Multiphase Flows, Cambridge University Press, 2011. 
[3] R. Scardovelli, S. Zaleski, Analytical relations connecting linear interfaces and volume fractions in rectangular grids, J. Comput. Phys. 164 (1) (2000) 228-237. doi:http://dx.doi.org/10.1006/jcph.2000.6567.

[4] X. Yang, A. J. James, Analytic relations for reconstructing piecewise linear interfaces in triangular and tetrahedral grids, J. Comput. Phys. 214 (1) (2006) 41-54. doi:http://dx.doi.org/10.1016/j.jcp.2005.09.002.

[5] J. López, J. Hernández, Analytical and geometrical tools for 3d volume of fluid methods in general grids, J. Comput. Phys. 227 (12) (2008) 5939-5948. doi:http://dx.doi.org/10.1016/j.jcp.2008.03.010.

[6] F. Ham, K. Mattson, G. Iaccarino, Accurate and stable finite volume operators for unstructured flow solvers, Annual Research Briefs, Center for Turbulence Research, Stanford University (2006) 243-261.

[7] B. J. Parker, D. L. Youngs, Two and three dimensional Eulerian simulation and fluid flow with material interfaces, Tech. Rep. 01/92, AWE (1992).

[8] K. Ito, T. Kunugi, H. Ohshima, T. Kawamura, Formulations and validations of a high-precision volume-of-fluid algorithm on nonorthogonal meshes for numerical simulations of gas entrainment phenomena, J. Nucl. Sci. Technol. 46 (4) (2009) 366-373. doi:10.1080/18811248.2007.9711542.

[9] L. Jofre, O. Lehmkuhl, J. Castro, A. Oliva, A 3-d volumeof-fluid advection method based on cell-vertex velocities for unstructured meshes, Comput. Fluids 94 (0) (2014) 14-29. doi:http://dx.doi.org/10.1016/j.compfluid.2014.02.001.

[10] J. E. Pilliod Jr., E. G. Puckett, Second-order accurate volume-of-fluid algorithms for tracking material interfaces, J. Comput. Phys. 199 (2) (2004) 465-502. doi:http://dx.doi.org/10.1016/j.jcp.2003.12.023.

[11] R. Scardovelli, S. Zaleski, Interface reconstruction with least-square fit and split Eulerian Lagrangian advection, Int. J. Numer. Meth. Fl. 41 (3) (2003) 251-274. doi:http://dx.doi.org/10.1002/fld.431. 
[12] E. Aulisa, S. Manservisi, R. Scardovelli, S. Zaleski, Interface reconstruction with least-squares fit and split advection in three-dimensional cartesian geometry, J. Comput. Phys. 225 (2) (2007) 2301-2319. doi:http://dx.doi.org/10.1016/j.jcp.2007.03.015.

[13] N. Ashgriz, T. Barbat, G. Wang, A computational Lagrangian-Eulerian advection remap for free surface flows, Int. J. Numer. Meth. Fl. 44 (1) (2004) 1-32. doi:10.1002/fld.620.

[14] S. J. Mosso, B. K. Schartz, D. B. Kothe, Recent enhancements of volume tracking algorithms for irregular grids., Tech. Rep. LA-CP-96-227, Los Alamos National Laboratory (1996).

[15] S. J. Cummins, M. M. Francois, D. B. Kothe, Estimating curvature from volume fractions, Comput. Struct. 83 (6-7) (2005) 425-434. doi:http://dx.doi.org/10.1016/j.compstruc.2004.08.017.

[16] M. Sussman, A second order coupled level set and volume-of-fluid method for computing growth and collapse of vapor bubbles, J. Comput. Phys. 187 (1) (2003) 110-136. doi:http://dx.doi.org/10.1016/S00219991(03)00087-1.

[17] M. M. Francois, S. J. Cummins, E. D. Dendy, D. B. Kothe, J. M. Sicilian, M. W. Williams, A balanced-force algorithm for continuous and sharp interfacial surface tension models within a volume tracking framework, J. Comput. Phys. 213 (1) (2006) 141-173. doi:http://dx.doi.org/10.1016/j.jcp.2005.08.004.

[18] G. Bornia, A. Cervone, S. Manservisi, R. Scardovelli, S. Zaleski, On the properties and limitations of the height function method in twodimensional cartesian geometry, J. Comput. Phys. 230 (4) (2011) 851-862. doi:http://dx.doi.org/10.1016/j.jcp.2010.11.029.

[19] P. A. Ferdowsi, M. Bussmann, Second-order accurate normals from 
height functions, J. Comput. Phys. 227 (22) (2008) 9293-9302. doi:http://dx.doi.org/10.1016/j.jcp.2008.07.014.

[20] M. M. Francois, B. K. Swartz, Interface curvature via volume fractions, heights, and mean values on nonuniform rectangular grids, J. Comput. Phys. 229 (3) (2010) 527-540. doi:http://dx.doi.org/10.1016/j.jcp.2009.10.022.

[21] K. Ito, T. Kunugi, S. Ohno, H. Kamide, H. Ohshima, A highprecision calculation method for interface normal and curvature on an unstructured grid, J. Comput. Phys. 273 (0) (2014) 38-53. doi:http://dx.doi.org/10.1016/j.jcp.2014.04.058.

[22] J. U. Brackbill, D. B. Kothe, C. Zemach, A continuum method for modeling surface tension, J. Comput. Phys. 100 (2) (1992) 335-354. doi:10.1016/0021-9991(92)90240-Y.

[23] K. Ito, T. Kunugi, H. Ohshima, T. Kawamura, A volumeconservative PLIC algorithm on three-dimensional fully unstructured meshes, Comput. Fluids 88 (0) (2013) 250-261. doi:http://dx.doi.org/10.1016/j.compfluid.2013.09.016.

[24] J. López, C. Zanzi, P. Gómez, R. Zamora, F. Faura, J. Hernández, An improved height function technique for computing interface curvature from volume fractions, Comput. Method. Appl. M. 198 (33-36) (2009) 25552564. doi:http://dx.doi.org/10.1016/j.cma.2009.03.007.

[25] J. López, J. Hernández, On reducing interface curvature computation errors in the height function technique, J. Comput. Phys. 229 (13) (2010) 48554868. doi:http://dx.doi.org/10.1016/j.jcp.2010.03.032.

[26] M. Owkes, O. Desjardins, A mesh-decoupled height function method for computing interface curvature, Journal of Computational Physics 281 (0) (2015) 285 - 300. doi:http://dx.doi.org/10.1016/j.jcp.2014.10.036. 
[27] S. Popinet, An accurate adaptive solver for surface-tension-driven interfacial flows, Journal of Computational Physics 228 (16) (2009) 5838 - 5866. doi:http://dx.doi.org/10.1016/j.jcp.2009.04.042.

URL http://www.sciencedirect.com/science/article/pii/S002199910900240X

[28] J. Hernández, J. López, P. Gómez, C. Zanzi, F. Faura, A new volume of fluid method in three dimensions-Part I: Multidimensional advection method with face-matched flux polyhedra, Int. J. Numer. Meth. Fl. 58 (8) (2008) 897-921. doi:10.1002/fld.1776.

[29] M. Herrmann, A balanced force refined level set grid method for two-phase flows on unstructured flow solver grids, J. Comput. Phys. 227 (4) (2008) 2674-2706. doi:10.1016/j.jcp.2007.11.002.

[30] J. Bonet, J. Peraire, An alternating digital tree (ADT) algorithm for 3d geometric searching and intersection problems, Int. J. Numer. Meth. Eng. 31 (1) (1991) 1-17. doi:10.1002/nme.1620310102.

[31] C. B. Ivey, P. Moin, Conservative volume of fluid advection method on unstructured grids in three dimensions, Annual Research Briefs, Center for Turbulence Research, Stanford University (2012) 179-192.

[32] V. L. Chenadec, H. Pitsch, A 3d unsplit forward/backward volume-of-fluid approach and coupling to the level set method, J. Comput. Phys. 233 (0) (2013) 10-33. doi:http://dx.doi.org/10.1016/j.jcp.2012.07.019.

[33] M. Owkes, O. Desjardins, A computational framework for conservative, three-dimensional, unsplit, geometric transport with application to the volume-of-fluid (VOF) method, J. Comput. Phys. 270 (0) (2014) 587-612. doi:http://dx.doi.org/10.1016/j.jcp.2014.04.022.

[34] M. B. Stephenson, H. N. Christiansen, A polyhedron clipping and capping algorithm and a display system for three dimensional finite element models, SIGGRAPH Comput. Graph. 9 (3) (1975) 1-16. doi:10.1145/988049.988050. 
[35] T. H. Cormen, C. E. Leiserson, R. L. Rivest, C. Stein, Introduction to Algorithms, Third Edition, The MIT Press, 2009.

[36] J. O'Rourke, Computational Geometry in C, Cambridge University Press, 1998. 